Gynecologic and

Obstetric Investigation

\title{
Low Back Pain in Pregnancy: Investigations, Management, and Role of Neuraxial Analgesia and Anaesthesia: A Systematic Review
}

\author{
Herman Sehmbi ${ }^{a}$ Rohan D'Souza ${ }^{b}$ Anuj Bhatia ${ }^{c}$ \\ ${ }^{a}$ Department of Anaesthesiology and Perioperative Medicine, University of Western Ontario, University Hospital, \\ LHSC, London, ON, 'bivision of Maternal and Fetal Medicine, Department of Obstetrics and Gynaecology, Mount \\ Sinai Hospital, and Institute of Health Policy Management and Evaluation, University of Toronto, and ${ }^{\mathrm{C}}$ Department \\ of Anaesthesia and Pain Management and Institute of Health Policy Management and Evaluation, University of \\ Toronto, and University Health Network-Toronto Western Hospital, Toronto, ON, Canada
}

\section{Keywords}

Low back pain $\cdot$ Pregnancy $\cdot$ Sciatica $\cdot$ Neuraxial .

Analgesia - Anaesthesia

\begin{abstract}
Background: Low back pain (LBP) is commonly experienced during pregnancy and is often poorly managed. There is much ambiguity in diagnostic work-up, appropriate management and decision-making regarding the use of neuraxial analgesia and anaesthesia during labour and delivery in these patients. This systematic review summarises the evidence regarding investigations, management strategies and considerations around performing neuraxial blocks for pregnant women with LBP. Methods: We searched 3 databases and reviewed literature concerning LBP in pregnancy with regards to diagnostic modalities, management strategies and use of neuraxial techniques for facilitating labour and delivery. Results: In all, we included 78 studies in this review, with 32 studies concerning diagnostic investigations, 56 studies involving management strategies, and 4 studies regarding the use of neuraxial techniques for labour and delivery. Summary: MRI is the safest investigative modality for LBP in pregnancy. Antenatal educational programmes, exercise and steroid injections into the epidural space or sacroil-
\end{abstract}

\section{KARGER}

(C) 2017 S. Karger AG, Basel

E-Mail karger@karger.com

www.karger.com/goi iac joints may help with pain management. Worsening neurological deficits, vertebral fractures and tumours may need surgical management. There is limited evidence on challenges of performing neuraxial blocks in the peripartum period for analgesia and anaesthesia, but there is a potential for increased risk of neurological complications in parturients with pre-existing neurological deficits.

(c) 2017 S. Karger AG, Basel

\section{Introduction}

Low back pain (LBP) is commonly experienced during pregnancy, affecting approximately 50\% of pregnant women [1]. It is associated with significant morbidity in pregnant and post-partum patients $[2,3]$ with persistent pain lasting 2 years in a tenth of the patients [3-7]. Despite its prevalence, LBP during pregnancy is often considered a normal phenomenon. This benign neglect can contribute to physical inactivity during pregnancy, resulting in a higher incidence of obstetric complications [8] and caesarean sections $[9,10]$. The epidemiology and the pathophysiology of LBP in pregnancy have been reviewed extensively $[11,12]$. However, there is still much ambiguity with regard to the definition of LBP in pregnancy, iden-

Anuj Bhatia, MBBS, MD, FRCA, FRCPC, FIPP, FFPMRCA, EDRA, CIPS Department of Anaesthesia and Pain Management and Institute of Health Policy Management and Evaluation, University of Toronto, University Health NetworkToronto Western Hospital, McL 2-405, 399 Bathurst St. Toronto, ON M5T 2S8 (Canada) E-Mail anuj.bhatia@uhn.ca 
tifying appropriate investigations, considering safe treatment options in pregnancy [13-15], and decision-making regarding the use of neuraxial analgesia and anaesthesia during labour and delivery $[16,17]$. These considerations tend to differ from those for LBP in non-pregnant individuals. Choice of imaging modalities for investigating LBP during pregnancy is affected by the desire to avoid ionizing radiation exposure to the fetus. Concerns regarding maternal and fetal well-being also entail the use of conservative treatment regimens [18].

The primary objective of this review was to determine clinically useful and safe diagnostic investigations and management strategies for LBP in pregnancy. The secondary objective was to evaluate evidence and make recommendations for neuraxial analgesia and anaesthesia during labour and delivery in pregnant women with LBP.

\section{Methods}

\section{Search Strategy and Study Selection}

We conducted a literature search using the OvidSP search platform in 3 bibliographic databases - Medline, Medline In-Process and Embase, using indexing terms related to pregnancy, LBP, and neuraxial techniques (online suppl. E-Table 1, see www.karger. com/doi/10.1159/000471764) to include articles indexed as of November 13,2015. The search was limited to human data in pregnant and post-partum patients and restricted to the English language.

\section{Criteria for Considering Studies for This Systematic Review}

Studies

We followed the Preferred Reporting Items for Systematic Reviews and Meta-Analyses recommendations during the preparation of this review [19]. We considered randomized controlled trials (RCTs), retrospective or prospective observational studies, case series or reports, conference proceedings and narrative reviews on investigative modalities, management strategies and the use of neuraxial (subarachnoid or epidural or combined spinal epidural analgesia/anaesthesia) techniques for pregnant women with LBP. We pre-specified eligibility criteria, which are as follows.

\section{Participants}

Pregnant subjects with new onset or worsening of pre-existing LBP of moderate-to-severe intensity (numerical rating score for pain of 4 or more on a $0-10$ scale) were included. An analysis from pain diagrams drawn by pregnant patients reveals the predominance of pain in either the lower lumbar area or around the sacroiliac joints (SIJ) [2]. While the former has been addressed as "pregnancy-related LBP" (PLBP), the latter has been termed as "pregnancy-related pelvic girdle pain" (PGP) [3]. While PLBP represents low lumbar back pain between the 12th rib and the gluteal fold, PGP represents pain experienced between the posterior iliac crest and the gluteal fold [20]. Considering that there is considerable overlap between the 2 and that many women present with both, for purposes of this review, we included both PLBP and PGP under the term LBP.
Investigations and Interventions

(1) Diagnostic investigations for LBP in pregnancy included fluoroscopy (X-ray), CT, MRI, ultrasonography and electrodiagnostic studies such as electromyography (EMG) and nerve conduction studies (NCS).

(2) Therapies for treatment of LBP in pregnancy included physical therapy, patient education and psychological therapies, complimentary treatments (mechanical supports, osteopathic manipulative treatment [OMT], water gymnastics, yoga, acupuncture and transcutaneous electrical nerve stimulation [TENS]) and interventional techniques including injections (injection of steroids with or without local anaesthetics into the epidural space, sacro-iliac joint and symphysis pubis) and miscellaneous interventions (kyphoplasty, vertebroplasty, and laminectomy).

(3) Neuraxial analgesia and anaesthesia techniques for facilitating labour and delivery in parturients with LBP included spinal, epidural and combined spinal-epidural techniques. In this section, we also included patients with previous spine surgery or spinal implants (such as spinal cord stimulators [SCS]), because LBP is common in this cohort.

\section{Comparator}

Data on comparator group(s), when available, was collected and reported.

\section{Outcomes}

For diagnostic modalities, we looked at whether the modality was able to determine an underlying cause for LBP. For interventions, we looked at the efficacy in terms of impact on patient-reported pain scores or reduction in analgesic requirements and impact on functional ability.

\section{Assessment of Bias}

Two authors (H.S. and R.D.) independently assessed the quality of included RCTs using the Cochrane Collaboration "Risk of Bias" tool for randomized trials [21]. Any disagreement was resolved through discussion or, if necessary, arbitration by a third reviewer (A.B.). The Cochrane tool assesses bias in the following domains: generation of the allocation sequence, allocation concealment, blinding of investigators and participants, blinding of outcome assessors, incomplete outcome data, selective outcome reporting and any other sources of bias. Each item is classified as low, unclear or high risk of bias. A decision to classify "overall bias" as low, unclear or high was made by the reviewers using the following method:

- High: any trial with a high risk of bias listed on 3 or more domains or significant methodological concerns that may have affected results.

- Unclear: any trial with a high risk of bias listed on 2 domains or moderate methodological concerns that may have affected study results.

- Low: any trial with a high risk of bias on none or 1 domain and with no significant methodological concerns that may have affected the study results.

\section{Data Collection}

Studies on pregnant and post-partum patients reporting on one or more of our outcomes of interest were included in this review. Reference data, populations, and outcomes were extracted from the articles into pre-specified tables using a standardized data extraction procedure by 2 of the authors (H.S. and A.B.). They ex-
418
Gynecol Obstet Invest 2017;82:417-436 DOI: $10.1159 / 000471764$
Sehmbi/D’Souza/Bhatia 
tracted information on studies' general characteristics (including design, number of arms and primary outcomes), participants (characteristics of the populations, sample size), interventions (diagnostic modality or therapeutic technique or neuraxial techniques), comparator (if any), parameters used for assessing efficacy of the intervention and summary of main outcomes.

\section{Results}

\section{Search Results}

The search results and study selection flowchart is reported in Figure 1. A total of 6,803 records were identified through database searching and 61 through citation tracking. After excluding duplicates and screening titles and abstracts, 78 studies were found to be relevant to the objectives of this review and these were included for synthesis and discussion. This included 32 RCTs, 2 non-randomized CTs, 3 review of literatures, 3 cohort studies, 2 prospective case series, 5 retrospective case series, 29 case reports, 1 letter to the editor and 1 conference proceeding.

\section{Risk of Bias Assessment}

The reviewers' consensus assessment of the risk is detailed in Figure 2. The overall risk of bias across the studies was moderate. Most studies adequately stated the methods used for randomization and allocation concealment barring 4 RCTs [22-25]. Blinding of participants and personnel was considered "unclear" in most studies barring "low risk" in only one study [26] and high risk in 3 other studies [27-29]. Theblinding of outcome assessors was judged "unclear risk" in 17 studies and "low risk" in 12 studies [26-36]. All trials barring one study [37] were judged at "low" or "unclear" risk of attrition bias and selective reporting. Most studies were determined to be at "unclear risk" of other bias mainly due to lack of adequate details regarding blinding or outcome assessment. Finally, the overall bias was adjudged as "low" in 15 studies and as "unclear" in the remaining 14 studies.

\section{a. Diagnostic Investigations for LBP during Pregnancy}

Of the 32 studies concerning diagnostic investigations, there were 3 prospective and 2 retrospective observational studies, 26 case reports, and 1 review of literature (Table 1). Most patients were investigated when the intensity of pain was moderate or severe, or associated with neurological compromise.

\section{Magnetic Resonance Imaging}

MRI was most commonly used modality (17 publications) for diagnosing the cause of LBP, especially when LBP was associated with neurological symptoms in the

LBP in Pregnancy: Role of Neuraxial

Analgesia and Anaesthesia

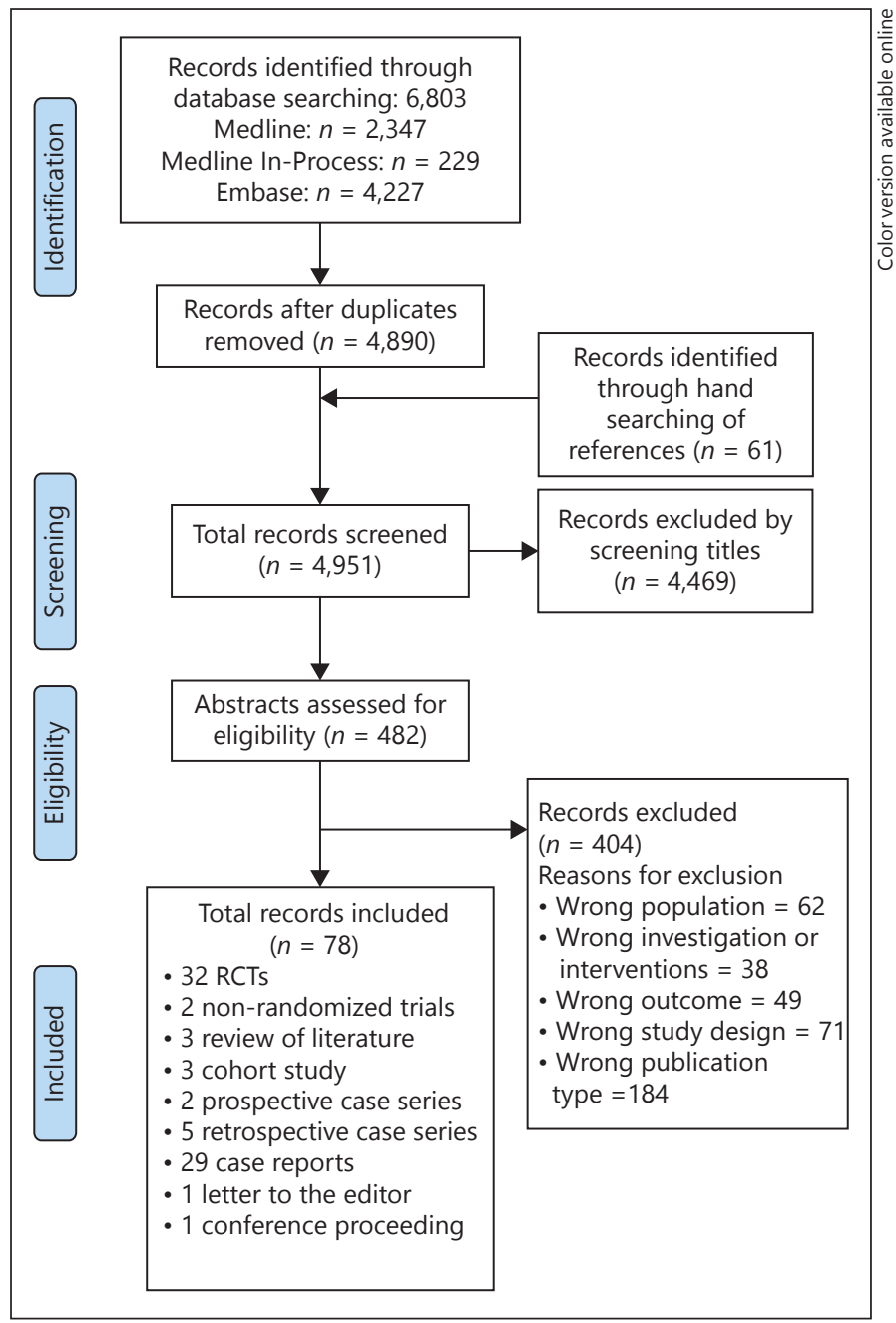

Fig. 1. PRISMA search strategy and selection of included articles.

lower limb such as radicular pain, paraplegia and/or cauda equina $[18,38-51]$. The underlying pathologies associated with these presentations included herniation of intervertebral discs $[18,38-46]$, vertebral hemangiomas [51], spinal body tumour [44], and tuberculosis [44] (Table 1). MRI was also used to investigate LBP in pregnant women with co-existing hip pain, with 3 studies reporting inflammation of the SIJ as the underlying cause [52-54] and 10 studies describing sacral fractures [5564].

\section{Imaging Modalities Involving the Use of Ionizing} Radiation (X-Ray and CT)

Six publications reported the use of CT scan for evaluating LBP during pregnancy or postpartum. The pathologies evaluated included vertebral collapse [47] and LBP

Gynecol Obstet Invest 2017;82:417-436 419 
Fig. 2. Risk of bias summary for included RCTs.

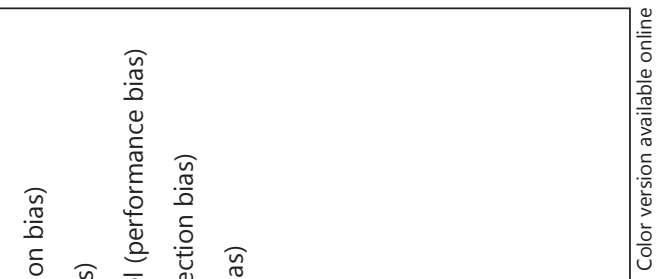

Bastianen et al., 2006

Eggen et al., 2012

Ekdahl et al., 2010

Elden et al., 2005

Elden et al., 2008

Garshabi et al., 2005

George et al., 2007

Guerreiro et al., 2004

Gutke et al., 2010

Haakstad et al., 2015

Haugland et al., 2006

Kalus et al., 2007

Kashanian et al., 2009

Keskin et al., 2012

Kihlstrand et al., 1999

Kluge et al., 2011

Kvorning et al., 2004

Licciardone et al., 2010

Lund et al., 2006

Martins et al., 2014

Mens et al., 2000

Miquelutti et al., 2013

Nilson-Wikmar et al., 2005

Ostgaard et al., 1994

Ostgaard et al., 1997

Schwerla et al., 2015

Stafne et al., 2012

Stuge et al., 2004

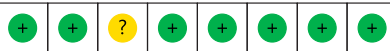

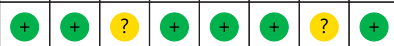

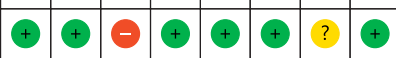

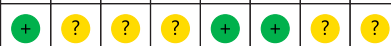

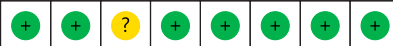

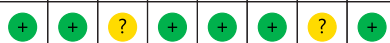

\begin{tabular}{l|l|l|l|l|l|l|l|}
\hline & $\odot$ & $?$ & $?$ & $?$ & $\odot$ & $?$ & $\odot$ \\
\hline
\end{tabular}

\begin{tabular}{ll|l|l|l|l|l|l|}
\hline & $?$ & $?$ & $?$ & $\odot$ & $\odot$ & $?$ & $?$
\end{tabular}

\begin{tabular}{l|l|l|l|l|l|l|l|}
\hline- & - & $?$ & $?$ & + & + & $?$ & $?$
\end{tabular}

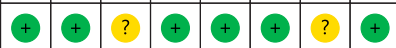

\begin{tabular}{l|l|l|l|l|l|l|l|}
\hline & $\odot$ & $?$ & $?$ & $\odot$ & $\odot$ & $?$ & $\odot$ \\
\hline
\end{tabular}

\begin{tabular}{ll|l|l|l|l|l|l}
\hline & $?$ & $?$ & $?$ & - & + & $?$ & ?
\end{tabular}

\begin{tabular}{ll|l|l|l|l|l|l|}
\hline & $?$ & $?$ & $?$ & + & $\odot$ & $?$ & $?$
\end{tabular}

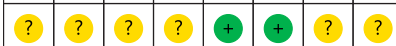

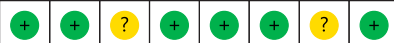

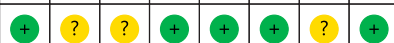

\begin{tabular}{lll|l|l|l|l|l|l|}
\hline & $?$ & $?$ & $?$ & $\odot$ & $\odot$ & $?$ & ? \\
\hline
\end{tabular}

\begin{tabular}{l|l|l|l|c|c|c|c|}
\hline & $?$ & $?$ & $\odot$ & $\odot$ & $\odot$ & $?$ & $\odot$ \\
\hline
\end{tabular}

$\begin{array}{lllllllllll}+ & ? & ? & + & + & + & + & +\end{array}$

\begin{tabular}{ll|l|l|l|l|l|l|}
\hline & $?$ & $?$ & $?$ & $\oplus$ & $\odot$ & $?$ & $?$
\end{tabular}

\begin{tabular}{ll|l|l|l|l|l|l|}
\hline & + & $?$ & $?$ & $?$ & $\odot$ & $?$ & $?$ \\
\hline
\end{tabular}

\begin{tabular}{|c|c|c|c|c|c|c|c|}
\hline$?$ & $?$ & $\odot$ & + & + & $\odot$ & $?$ & $?$ \\
\hline
\end{tabular}

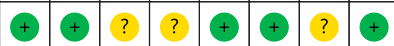

○

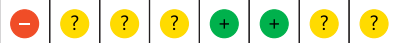

\begin{tabular}{|c|c|c|c|c|c|c|c|}
\hline & $?$ & $?$ & $?$ & + & $\odot$ & $?$ & $?$ \\
\hline
\end{tabular}

\begin{tabular}{|c|c|c|c|c|c|c|c|}
\hline+ & $\odot$ & $?$ & $?$ & $\odot$ & $\odot$ & $?$ & $\odot$ \\
\hline$\odot$ & $\odot$ & $?$ & $?$ & $\odot$ & $\odot$ & $?$ & $\odot$ \\
\hline$\odot$ & $?$ & $\odot$ & $\odot$ & $\odot$ & $\odot$ & $?$ & $?$ \\
\hline
\end{tabular}

Sehmbi/D’Souza/Bhatia 
Table 1. Summary of studies of diagnostic investigations performed to evaluate low back pain in pregnancy (classified by pathology)

\begin{tabular}{|c|c|c|c|}
\hline Study author, year, type, number of patients & Assessment modality & Reason for investigation & Finding on investigation \\
\hline \multicolumn{4}{|l|}{ Pelvic floor dysfunction } \\
\hline Arab et al. [67], 2010, CS-R, $n=40$ & Trans-abdominal pelvic floor US & $\begin{array}{l}\text { To evaluate the role of pelvic muscle } \\
\text { dysfunction in LBP }\end{array}$ & $\begin{array}{l}\text { Patients with LBP had pelvic floor muscle } \\
\text { dysfunction }\end{array}$ \\
\hline \multicolumn{4}{|l|}{ Disc herniation } \\
\hline Abou-Shameh et al. [38], 2005, CR, $n=1$ & MRI & LBP and leg pain & Disc herniation \\
\hline Al-Areibi et al. [39], 2007, CR, $n=1$ & MRI & LBP, leg pain and cauda equina syndrome & Disc herniation \\
\hline Brown et al. [40], 2001, CR, $n=3$ & MRI & LBP, leg pain and/or cauda equina syndrome & Disc herniation \\
\hline Brown et al. [41], 2004, CR, $n=1$ & MRI & LBP, leg pain and cauda equina syndrome & Disc herniation \\
\hline Darwich et al. [42], 2009, CR, $n=1$ & MRI & Leg pain & Disc herniation \\
\hline Garmel et al. [43], 1995, CR, $n=3$ & MRI & LBP, leg pain and cauda equina syndrome & Disc herniation \\
\hline Han et al. [44], 2010, CS-R, $n=10$ & MRI & LBP and/or paraparesis & $\begin{array}{l}\text { Disc herniation }(6 / 10 \text { patients }) \\
\text { Spinal/vertebral body tumor }(3 / 10 \text { patients) } \\
\text { Spinal tuberculosis ( } 1 / 10 \text { patients })\end{array}$ \\
\hline Kim et al. [45], 2007, CR, $n=1$ & MRI & LBP, leg weakness and numbness & Disc herniation \\
\hline LaBan et al. [46], 1995, CS-R, $n=6$ & $\begin{array}{l}\text { MRI } \\
\text { EMG }\end{array}$ & LBP and leg pain & $\begin{array}{l}\text { Disc herniation (MRI - } 6 \text { patients) } \\
\text { Radiculopathy (EMG - } 4 \text { patients) }\end{array}$ \\
\hline Lee et al. [18], 2011, CR, $n=1$ & MRI & LBP, leg pain and numbness & Disc herniation \\
\hline \multicolumn{4}{|l|}{ Vertebral pathology } \\
\hline Bayram et al. [47], 2006, CR, $n=1$ & Lumbar X-rays, MRI & LBP post-partum & Vertebral fracture \\
\hline Hsieh et al. [50], 2004, CR, $n=1$ & MRI & LBP, numbness, weakness, cauda equina & $\begin{array}{l}\text { Collapsed } 11 \text { th thoracic vertebra and } \\
\text { spinal tumor }\end{array}$ \\
\hline Kim et al. [48], 2010, CR, $n=1$ & MRI & LBP & Multiple vertebral compression fractures \\
\hline Moles et al. [51], 2014, ROL, $n=27(2+25)$ & MRI & Paraplegia, upper back pain, cauda equina & Vertebral hemangioma \\
\hline Ozdemir et al. [49], 2015, CR , $n=2$ & MRI & Postpartum LBP & Vertebral fractures \\
\hline \multicolumn{4}{|l|}{ Sacroilitis } \\
\hline Egerman et al. [52], 1995, CR, $n=1$ & Pelvic X-rays, CT and MRI & Sacroilitis causing hip and pelvic pain & Sacroilitis \\
\hline Mulvey et al. [53], 2008, CR, $n=1$ & MRI & Back and thigh pain & Sacroilitis \\
\hline Park et al. [54], 2013, CR, $n=1$ & MRI, CT & Hip pain & Sacroilitis \\
\hline \multicolumn{4}{|l|}{ Sacral fractures } \\
\hline Giannoulis et al. [55], 2015, CR, $n=1$ & MRI & LBP & Sacral fracture \\
\hline Goeb et al. [56], 2015, CR, $n=1$ & MRI & LBP & Sacral fracture \\
\hline Karatas et al. [57], 2008, CR, $n=1$ & Pelvic X-rays, MRI and CT & LBP and buttock pain & Sacral fracture \\
\hline Onur et al. [58], 2011, CR, $n=1$ & MRI and CT & LBP and buttock pain & Sacral fracture \\
\hline Ozturk et al. [59], 2013, CR, $n=1$ & MRI & Postpartum LBP and hip pain & Sacral fractures \\
\hline Park et al. [60], 2013, CR, $n=1$ & MRI & Postpartum LBP and leg pain & Sacral fractures \\
\hline Pishnamaz et al. [61], 2012, CR, $n=1$ & MRI & LBP & Sacral fracture \\
\hline Rousiere et al. [62], 2001, CR, $n=1$ & MRI & LBP & Sacral fracture \\
\hline Sansone et al. [63], 2013, CR, $n=1$ & MRI & LBP & Sacral fracture \\
\hline Thein et al. [64], 2009, CR, $n=1$ & MRI & LBP and leg pain & Sacral fracture \\
\hline
\end{tabular}

LBP in Pregnancy: Role of Neuraxial Analgesia and Anaesthesia 
Table 1. (continued)

\begin{tabular}{|c|c|c|c|}
\hline Study author, year, type, number of patients & Assessment modality & Reason for investigation & Finding on investigation \\
\hline \multicolumn{4}{|l|}{ Miscellaneous } \\
\hline Damen et al. [66], 2001, CO, $n=16$ & $\begin{array}{l}\text { US (Doppler imaging of } \\
\text { vibrations from SIJ) }\end{array}$ & $\begin{array}{l}\text { To study the role asymmetry of SIJ laxity with } \\
\text { pregnancy related pelvic pain }\end{array}$ & Asymmetry of SIJ laxity \\
\hline Chan et al. [160], 2002, CS-P, $n=105$ & MRI & $\begin{array}{l}\text { To study the relationship of intervertebral disc } \\
\text { abnormality and signal changes of the uterine } \\
\text { cervix on MRI }\end{array}$ & $\begin{array}{l}\text { Significant correlation of high signal } \\
\text { intensity in the uterine cervix and LBP } \\
\text { suggested that soft tissue laxity may be a } \\
\text { more important cause of back pain in } \\
\text { pregnancy than disk bulge or prolapse }\end{array}$ \\
\hline Hasegawa et al. [65], 1999, CR, $n=1$ & $\begin{array}{l}\text { Pelvic X-rays, CT, MRI, bone } \\
\text { scintigraphy }\end{array}$ & Posterior pelvic girdle pain & Slight irregularity of right SI joint (MRI) \\
\hline
\end{tabular}

CR, case report; CS-P, case series-prospective; CS-R, case series-retrospective; CO, cohort study; ROL, review of literature; US, ultrasound; MRI, magnetic resonance imaging; EMG, electromyography; LBP, low back pain; SIJ, sacroiliac joint.

associated with hip, pelvic or buttock pain $[52,54,57,58$, 65]. While some of these scans were done during pregnancy $[52,65]$, most were done during the postpartum period including a therapeutic CT-guided hip joint aspiration in a postpartum woman with buttock pain [54].

\section{Ultrasound}

We identified one study that used Doppler ultrasound to identify SIJ laxity [66] and another prospective study that utilized trans-abdominal pelvic floor ultrasound to identify pelvic floor muscle dysfunction in parturients with LBP [67].

\section{Electro-Diagnostic Studies (EMG and NCS)}

One study reported the use of EMG in conjunction with MRI for evaluating4 pregnant patients with lumbar radiculopathy in the third trimester [46]. No publications reporting the use of NCS were identified.

\section{b. Therapies for LBP in Pregnancy}

There were 32 RCTs among the 57 publications outlining management strategies for LBP in pregnancy ( $\mathrm{Ta}-$ ble 2). The following therapies were identified:

\section{Multimodal Management Including Patient}

Education, Exercise, and Psychological Therapies

Six RCTs and one non-randomized trial studied multimodal management (education, exercise, and/or others) often as integrated antenatal classes for management of LBP in pregnancy (Table 2a). Bastiaenen et al. [68] studied the effectiveness of a tailor-made program modifying biopsychosocial factors (i.e., targeting patients' anxieties) in women with PLBP, and found it to be effective in improving the limitations in activities as compared to the control group (usual care). While 3 other studies reported better analgesic and functional outcomes when compared with the standard care for $\operatorname{LBP}[25,69,70]$, the rest reported no difference $[24,37,71]$.

\section{Physical Therapy and Exercise}

Conservative measures such as exercises were evaluated in 14 RCTs, one non-randomized trial and one case series (Table 2b). Seven studies reported benefit [29, 30, $32,70,72-74]$, while the rest did not $[23,27,28,34,75-$ 78]. Similarly, PGP was reported to improve with pelvic tilt [73] and stabilization exercises [29], but not with truncal exercises [28]. Three studies did not show the benefit of exercise with PGP $[27,77,78]$.

\section{Complementary and Alternative Medicine}

Eleven RCTs, 2 cohort studies and 2 case reports evaluated the role of complementary therapies (Table 2c). In a cohort study, the use of pelvic belts was found to reduce SIJ mobility, thereby providing relief from LBP [79]. In one RCT, support devices (BellyBra ${ }^{\circledR}$, Tubigrip ${ }^{\circledR}$ ) were found to be effective in reducing PLBP [80]. OMT reduced LBP and the progression of back-specific dysfunction in pregnancy and postpartum in 3 studies $[26,81$, 82 ]. Water gymnastics [35] and yoga [83] were also found to be effective in reducing pain intensity.

Two case reports $[84,85]$ and 6 RCTs studied the role of acupuncture [22, 32, 33, 36, 86, 87]. Two of these RCTs demonstrated analgesic benefit when compared to controls $[22,36]$. Acupuncture, as an adjunct to standard treatment, was found to be better than standard treatment alone for reduction of pain and improving the ability to perform daily activities in pregnancy-related PGP $[32,33]$. However, timing of acupuncture during preg- 
Table 2. Summary of management strategies of LBP in pregnancy

a. Multimodal management including patient education, exercise, and psychological therapies

\begin{tabular}{|c|c|c|c|c|}
\hline Study, year, type, number of patients & $\begin{array}{l}\text { Clinical } \\
\text { presentation }\end{array}$ & Intervention (I) and comparator (C) & Assessment parameters & Outcomes \\
\hline George et al. [70], 2013, RCT, $n=169$ & LBP, PGP & $\begin{array}{l}\text { I: multimodal management (manual therapy, } \\
\text { exercise and education) } \\
\text { C: standard obstetric care }\end{array}$ & $\begin{array}{l}\text { NRS for pain and the Quebec } \\
\text { Disability Questionnaire }\end{array}$ & $\begin{array}{l}\text { Multimodal } \\
\text { management better } \\
\text { than standard obstetric } \\
\text { care }\end{array}$ \\
\hline Haugland et al. [37], 2006, RCT, $n=569$ & PGP & $\begin{array}{l}\text { I: education program that consisted of information, } \\
\text { ergonomics, exercises, pain management, advice } \\
\text { C: routine prenatal care }\end{array}$ & $\begin{array}{l}\text { Incidence of PGP during and } \\
\text { after pregnancy, and VAS }\end{array}$ & $\begin{array}{l}\text { No significant } \\
\text { differences between } \\
\text { the two groups in } \\
\text { incidence of back pain } \\
\text { during pregnancy and } \\
\text { post-partum }\end{array}$ \\
\hline Mantle et al. [69], 1981, NRCT, $n=208$ & LBP & $\begin{array}{l}\text { I: back care classes and antenatal classes } \\
\text { C: routine antenatal classes }\end{array}$ & Custom questionnaires & $\begin{array}{l}\text { Significantly less } \\
\text { "troublesome" and } \\
\text { "severe" backache later } \\
\text { during the pregnancy } \\
\text { with back care and } \\
\text { antenatal classes }\end{array}$ \\
\hline Miquelutti et al. [71], 2013, RCT, $n=197$ & LBP, PGP & $\begin{array}{l}\text { I: antenatal classes (exercises, educational activities } \\
\text { and instructions on exercises) } \\
\text { C: no antenatal classes }\end{array}$ & $\begin{array}{l}\text { Evaluated urinary incontinence, } \\
\text { lumbopelvic pain, physical activity, } \\
\text { and anxiety }\end{array}$ & $\begin{array}{l}\text { No difference was } \\
\text { found between the } \\
\text { groups regarding to } \\
\text { lumbopelvic pain }\end{array}$ \\
\hline Ostgaard et al. [24], 1994, RCT, $n=407$ & LBP, PGP & $\begin{array}{l}\text { I: } 2 \text { educational sessions or individualized classes } \\
\text { C: routine care }\end{array}$ & $\begin{array}{l}\text { Pain incidence and intensity, sick } \\
\text { leaves }\end{array}$ & $\begin{array}{l}\text { No difference in } \\
\text { incidence or intensity } \\
\text { of pain, but reduced } \\
\text { sick days with routine } \\
\text { care }\end{array}$ \\
\hline Ostgaard et al. [25], 1997, RCT, $n=362$ & LBP, PGP & $\begin{array}{l}\text { I: } 2 \text { educational sessions or } 5 \text { individualized classes } \\
\text { C: routine care }\end{array}$ & $\begin{array}{l}\text { Pain incidence and intensity during } \\
\text { pregnancy and post-partum }\end{array}$ & $\begin{array}{l}\text { Physiotherapy and } \\
\text { patient education } \\
\text { reduced back pain in } \\
\text { affected women }\end{array}$ \\
\hline Bastiaenen et al. [68], 2006, RCT, $n=126$ & LBP, PGP & $\begin{array}{l}\text { I: individualized program with biopsychosocial } \\
\text { factors } \\
\text { C: usual care based on a pain contingent basis }\end{array}$ & $\begin{array}{l}\text { LBP and PGP using Roland-Morris } \\
\text { Disability Questionnaire score }\end{array}$ & $\begin{array}{l}\text { Reduction in disability } \\
\text { with individualized } \\
\text { program }\end{array}$ \\
\hline
\end{tabular}

\section{b. Physical therapy}

\begin{tabular}{|c|c|c|c|}
\hline Study, year, type, number of patients & Intervention (I) and comparator (C) & Assessment parameters & Outcomes \\
\hline \multicolumn{4}{|l|}{$L B P$} \\
\hline $\begin{array}{l}\text { Akmese et al. [30], 2014, RCT, } \\
n=66\end{array}$ & $\begin{array}{l}\text { I: progressive muscle relaxation and } \\
\text { music therapy (LBP) } \\
\text { C: lying down }\end{array}$ & $\begin{array}{l}\text { Physical and psychological outcomes } \\
\text { studied VAS for pain; short form } 36 \text { for } \\
\text { quality of life }\end{array}$ & $\begin{array}{l}\text { Significant improvement in physical } \\
\text { and psychological outcomes at } 4 \text { and } \\
8 \text { weeks with muscle relaxation and } \\
\text { music therapy }\end{array}$ \\
\hline $\begin{array}{l}\text { Dumas et al. [75], 1995, NRCT, } \\
n=65\end{array}$ & $\begin{array}{l}\text { I: } 27 \text { were enrolled in exercise classes } \\
\text { C: } 38 \text { acted as sedentary controls }\end{array}$ & Custom questionnaires & $\begin{array}{l}\text { No significant difference between } \\
\text { intervention and comparator }\end{array}$ \\
\hline $\begin{array}{l}\text { Garshasbi et al. [72], 2005, RCT, } \\
n=212\end{array}$ & $\begin{array}{l}\text { I: exercise } \\
\text { C: no exercise }\end{array}$ & Custom questionnaires & $\begin{array}{l}\text { Significant reduction in the intensity } \\
\text { of LBP and flexibility in exercise } \\
\text { group }\end{array}$ \\
\hline $\begin{array}{l}\text { Kashanian et al. [76], 2009, RCT, } \\
n=30\end{array}$ & $\begin{array}{l}\text { I: regular exercise program } \\
\text { C: routine prenatal care }\end{array}$ & Severity on VAS & No significant differences \\
\hline \multicolumn{4}{|l|}{$P G P$} \\
\hline $\begin{array}{l}\text { Andrews et al. [73], 1994, CS, } \\
n=25\end{array}$ & $\begin{array}{l}\text { Pelvic tilt exercises for ligament pain } \\
\text { (pelvic pain) }\end{array}$ & $\begin{array}{l}\text { McGill pain questionnaire } \\
\text { Home recording card }\end{array}$ & Effective in reducing pelvic pain \\
\hline $\begin{array}{l}\text { Elden et al. [32], 2005, RCT, } \\
n=386\end{array}$ & $\begin{array}{l}\text { I: standard treatment (ST) combined } \\
\text { with acupuncture and ST combined with } \\
\text { stabilizing exercises } \\
\text { C: standard treatment }\end{array}$ & VAS for pain & $\begin{array}{l}\text { Acupuncture and stabilising } \\
\text { exercises constitute efficient } \\
\text { complements to standard treatment }\end{array}$ \\
\hline
\end{tabular}

LBP in Pregnancy: Role of Neuraxial Analgesia and Anaesthesia 
Table 2b. (continued)

\begin{tabular}{|c|c|c|c|}
\hline Study, year, type, number of patients & Intervention (I) and comparator (C) & Assessment parameters & Outcomes \\
\hline $\begin{array}{l}\text { Elden et al. [161], 2008, RCT, } \\
n=386\end{array}$ & $\begin{array}{l}\text { I: ST combined with acupuncture and ST } \\
\text { combined with stabilizing exercises } \\
\text { C: standard treatment }\end{array}$ & Adverse effects of acupuncture & $\begin{array}{l}\text { Minimal adverse effects of } \\
\text { acupuncture (efficacy studied in } \\
\text { earlier paper [31]) }\end{array}$ \\
\hline $\begin{array}{l}\text { Gutke et al. [34], 2010, RCT, } \\
n=88\end{array}$ & $\begin{array}{l}\text { I: stabilization exercises } \\
\text { C: no exercises }\end{array}$ & Oswestry disability Index & No significant difference \\
\hline $\begin{array}{l}\text { Mens et al. [28], 2000, RCT, } n=44 \\
\text { post-partum patients }\end{array}$ & $\begin{array}{l}\text { I: diagonal trunk exercise or longitudinal } \\
\text { trunk exercise } \\
\mathrm{C}: \text { no exercise }\end{array}$ & $\begin{array}{l}\text { Nottingham health profile, posterior pelvic } \\
\text { pain provocation test, and a radiographic } \\
\text { examination }\end{array}$ & $\begin{array}{l}\text { No significant differences between } \\
\text { groups }\end{array}$ \\
\hline $\begin{array}{l}\text { Nilsson-Wikmar et al. [23], 2005, RCT, } \\
n=118\end{array}$ & $\begin{array}{l}\text { I: } 1 \text {. information and pelvic belt } 2 \text {. home } \\
\text { exercises } 3 \text {. in clinic exercises }\end{array}$ & $\begin{array}{l}\text { VAS, pain drawing, and disability rating } \\
\text { index }\end{array}$ & $\begin{array}{l}\text { No significant differences between } \\
\text { groups }\end{array}$ \\
\hline $\begin{array}{l}\text { Stuge et al. [29], 2004, RCT, } n=81 \text {, } \\
\text { post-partum patients }\end{array}$ & $\begin{array}{l}\text { I: stabilization exercises } \\
\text { C: no stabilization exercises }\end{array}$ & $\begin{array}{l}\text { Baseline and } 1 \text { year pain, functional status } \\
\text { and quality of life }\end{array}$ & $\begin{array}{l}\text { Stabilization exercises effective in } \\
\text { reducing pain, improving functional } \\
\text { status, and improving health-related } \\
\text { quality of life }\end{array}$ \\
\hline \multicolumn{4}{|l|}{$L B P$ and $P G P$} \\
\hline Eggen et al. [27], 2012, RCT, $n=257$ & $\begin{array}{l}\text { I: group based exercise program } \\
\text { C: none }\end{array}$ & Short-Form Health Survey (SF-8) & $\begin{array}{l}\text { No benefit of exercise program on the } \\
\text { prevalence of PGP or LBP }\end{array}$ \\
\hline George et al. [70], 2013, RCT, $n=169$ & $\begin{array}{l}\text { I: multimodal management (manual therapy, } \\
\text { exercise and education) } \\
\text { C: standard obstetric care }\end{array}$ & $\begin{array}{l}\text { Numerical Rating Scale for pain and the } \\
\text { Quebec Disability Questionnaire }\end{array}$ & $\begin{array}{l}\text { Combination of manual therapy, } \\
\text { exercise, and education better than } \\
\text { control }\end{array}$ \\
\hline $\begin{array}{l}\text { Haakstad et al. [77], 2015, RCT, } \\
n=105\end{array}$ & $\begin{array}{l}\text { I: regular exercise program } \\
\text { C: no exercises }\end{array}$ & $\begin{array}{l}\text { Number of patients reporting pain after } \\
\text { intervention and post-partum }\end{array}$ & No differences between groups \\
\hline Kluge et al. [74], 2011, RCT, $n=50$ & $\begin{array}{l}\text { I: regular exercise program } \\
\text { C: no exercises }\end{array}$ & $\begin{array}{l}\text { Numerical Rating Scale for pain, } \\
\text { Roland-Morris Disability Questionnaire }\end{array}$ & $\begin{array}{l}\text { Decreased back pain intensity and } \\
\text { increased functional ability with } \\
\text { regular exercise program }\end{array}$ \\
\hline Stafne et al. [78], 2012, RCT, $n=855$ & $\begin{array}{l}\text { I: regular exercise program } \\
\text { C: no exercises }\end{array}$ & $\begin{array}{l}\text { Lumbopelvic pain using } \\
\text { self-reported measures }\end{array}$ & $\begin{array}{l}\text { No significant difference between } \\
\text { groups }\end{array}$ \\
\hline
\end{tabular}

c. Complementary and alternative medicine (CAM) therapies

\begin{tabular}{|c|c|c|c|}
\hline Study, year, type, number of patients & Intervention (I) and comparator (C) & Assessment parameters & Outcomes \\
\hline \multicolumn{4}{|l|}{ Mechanical support } \\
\hline Mens et al. [79], 2006, CO, $n=25$ & Application of a pelvic belt for pelvic pain & $\begin{array}{l}\text { SIJ laxity as assessed by Doppler } \\
\text { imaging }\end{array}$ & $\begin{array}{l}\text { Pelvic belt significantly decreased } \\
\text { mobility of the sacroiliac joints }\end{array}$ \\
\hline Kalus et al. [80], 2008, RCT, $n=115$ & $\begin{array}{l}\text { I : BellyBra } \\
\text { C: Tubigrip }\end{array}$ & $\begin{array}{l}\text { Pain severity and physical activity, and } \\
\text { satisfaction with life }\end{array}$ & $\begin{array}{l}\text { Both BellyBra and Tubigrip were } \\
\text { associated with a reduction in the severity } \\
\text { of pregnancy-related low back pain }\end{array}$ \\
\hline \multicolumn{4}{|l|}{$O M T$} \\
\hline $\begin{array}{l}\text { Licciardone et al. [26], 2010, RCT, } \\
n=144\end{array}$ & $\begin{array}{l}\text { I 1: usual obstetric care and OMT } \\
\text { I 2: usual obstetric care and sham ultrasound } \\
\text { treatment } \\
\text { C: usual obstetric care only }\end{array}$ & $\begin{array}{l}\text { Pain levels and Roland-Morris Disability } \\
\text { Questionnaire }\end{array}$ & $\begin{array}{l}\text { OMT slows or halts the deterioration of } \\
\text { back-specific functioning during the } \\
\text { third trimester of pregnancy }\end{array}$ \\
\hline $\begin{array}{l}\text { Peterson et al. [162], 2014, CO, } \\
n=115\end{array}$ & Chiropractic treatment & $\begin{array}{l}\text { Patients' global impression of change } \\
\text { (GPGIC), numerical rating scale (pain), } \\
\text { Oswestry Disability Index }\end{array}$ & $\begin{array}{l}\text { Significant reduction in NRS and } \\
\text { Oswestry scores }\end{array}$ \\
\hline $\begin{array}{l}\text { Schwerla et al. [82], 2015, RCT, } \\
n=80\end{array}$ & $\begin{array}{l}\text { I: OMT } \\
\text { C: usual obstetric care only }\end{array}$ & $\begin{array}{l}\text { Pain intensity and Oswestry Disability } \\
\text { Index }\end{array}$ & $\begin{array}{l}\text { OMT produced clinically relevant } \\
\text { reduction in pain intensity and } \\
\text { functional disability in postpartum LBP }\end{array}$ \\
\hline \multicolumn{4}{|l|}{ Water gymnastics } \\
\hline $\begin{array}{l}\text { Kihlstrand et al. [35], 1999, RCT, } \\
n=129\end{array}$ & $\begin{array}{l}\text { I: water gymnastics } \\
\text { C: none }\end{array}$ & $\begin{array}{l}\text { Questionnaires evaluating the intensity of } \\
\text { pain }\end{array}$ & $\begin{array}{l}\text { Reduction of pain intensity and sick } \\
\text { leaves with water intervention }\end{array}$ \\
\hline \multicolumn{4}{|l|}{ Yoga } \\
\hline $\begin{array}{l}\text { Martins et al. [83], 2014, RCT, } \\
n=60\end{array}$ & $\begin{array}{l}\text { I: yoga exercises } \\
\text { C: postural orientation }\end{array}$ & Lumbopelvic pain using VAS & Reduced pain intensity in yoga group \\
\hline
\end{tabular}


Table 2c. (continued)

\begin{tabular}{|c|c|c|c|}
\hline Study, year, type, number of patients & Intervention (I) and comparator (C) & Assessment parameters & Outcomes \\
\hline \multicolumn{4}{|l|}{ Acupuncture } \\
\hline Cummings [84], 2003, CR, $n=1$ & Acupuncture for back pain in pregnancy & Patient reported pain measures & Successful management of back pain \\
\hline Ekdahl et al. [86], 2010, RCT, $n=40$ & $\begin{array}{l}\text { I 1: acupuncture at } 20 \text { weeks } \\
\text { I 2: acupuncture at } 26 \text { weeks }\end{array}$ & $\begin{array}{l}\text { VAS, short form McGill Questionnaire, } \\
\text { short form 36, telephone interviews }\end{array}$ & $\begin{array}{l}\text { Pain relief in both groups, gr } 2 \text { showed } \\
\text { improvement in SF } 36 \text { despite less } \\
\text { physical activities }\end{array}$ \\
\hline Elden et al. [32], 2005, RCT, $n=386$ & $\begin{array}{l}\text { Standard treatment (ST) vs. ST plus } \\
\text { acupuncture vs. ST plus stabilizing exercises }\end{array}$ & $\begin{array}{l}\text { Pelvic girdle pain using: } \\
\text { VAS }\end{array}$ & $\begin{array}{l}\text { Acupuncture and stabilising exercises } \\
\text { constitute efficient complements to } \\
\text { standard treatment }\end{array}$ \\
\hline Elden et al. [33], 2008, RCT, $n=115$ & $\begin{array}{l}\text { I: penetrating acupuncture } \\
\text { C: non-penetrating sham acupuncture }\end{array}$ & $\begin{array}{l}\text { Pain, sick leave, functional status, } \\
\text { health-related quality of life and recovery } \\
\text { of severity of PGP }\end{array}$ & $\begin{array}{l}\text { No significant difference on pain or sick } \\
\text { leaves, some improvement in } \\
\text { performing daily activities }\end{array}$ \\
\hline Forrester et al. [85], 2003, CR, $n=1$ & Acupuncture for back pain in pregnancy & & Successful management \\
\hline $\begin{array}{l}\text { Guerreiro et al. [22], 2004, RCT, } \\
n=61\end{array}$ & $\begin{array}{l}\text { I: acupuncture } \\
\text { C: control group }\end{array}$ & $\begin{array}{l}\text { NRS, capacity to work/mobilize, analgesic } \\
\text { use }\end{array}$ & $\begin{array}{l}\text { Improvement in NRS, ability to } \\
\text { ambulate and reduction of analgesic } \\
\text { use in acupuncture group }\end{array}$ \\
\hline $\begin{array}{l}\text { Kvorning et al. [36], 2004, RCT, } \\
n=72\end{array}$ & $\begin{array}{l}\text { I: traditional acupuncture and } \\
\text { co-interventions } \\
\text { C: co-interventions (physiotherapy and } \\
\text { analgesics) }\end{array}$ & VAS & $\begin{array}{l}\text { Reduction in VAS in acupuncture } \\
\text { group }\end{array}$ \\
\hline Lund et al. [87], 2006, RCT, $n=47$ & $\begin{array}{l}\text { I: superficial acupuncture } \\
\text { C: deep acupuncture }\end{array}$ & VAS at rest or activity & $\begin{array}{l}\text { No significant differences were } \\
\text { observed }\end{array}$ \\
\hline \multicolumn{4}{|l|}{ TENS } \\
\hline Keskin et al. [88], 2012, RCT, $n=79$ & $\begin{array}{l}\text { I1: exercise, } \\
\text { I2: acetaminophen } \\
\text { I3: TENS } \\
\text { C: control (no treatment) }\end{array}$ & VAS and RMDQ after 3 weeks of therapy & $\begin{array}{l}\text { All intervention groups fared } \\
\text { significantly better than control. } \\
\text { TENS was both effective and safe }\end{array}$ \\
\hline
\end{tabular}

\section{d. Interventional or surgical management strategies}

\begin{tabular}{|c|c|c|c|}
\hline Study author, year, type, number of patients & Finding on investigation & Treatment strategy & Outcome \\
\hline \multicolumn{4}{|l|}{ Disc herniation } \\
\hline Abou-Shameh et al. [38], 2005, CR, $n=1$ & Disc herniation & Discectomy & $\begin{array}{l}\text { Resolution of leg weakness and pain } \\
\text { within one day of surgery }\end{array}$ \\
\hline Al-Areibi et al. [39], 2007, CR, $n=1$ & Disc herniation & Emergent delivery and lumbar discectomy & $\begin{array}{l}\text { Complete motor and sensory function of } \\
\text { the lower limbs, but residual bladder } \\
\text { dysfunction by discharge }\end{array}$ \\
\hline Brown et al. [40], 2001, CR, $n=3$ & Disc herniation & $\begin{array}{l}\text { ESI followed by laminectomy and } \\
\text { discectomy }\end{array}$ & $\begin{array}{l}\text { Improvement of pain in all cases, partial/ } \\
\text { complete recovery of neurological deficits }\end{array}$ \\
\hline Brown et al. [41], 2004, CR, $n=1$ & Disc herniation & $\begin{array}{l}\text { ESI followed by emergent delivery and } \\
\text { lumbar discectomy }\end{array}$ & $\begin{array}{l}\text { Improvement of pain and near complete } \\
\text { recovery of neurological deficits }\end{array}$ \\
\hline Darwich et al. [42], 2009, CR, $n=1$ & Disc herniation & $\begin{array}{l}\text { ESI followed by micro-discectomy } \\
\text { post-partum }\end{array}$ & Complete improvement of pain \\
\hline Garmel et al. [43], 1995, CR, $n=3$ & Disc herniation & Laminectomy and/or discectomy & $\begin{array}{l}\text { Improvement of pain and partial recovery } \\
\text { of neurological deficits }\end{array}$ \\
\hline Han et al. [44], 2010, CS-R, $n=10$ & $\begin{array}{l}\text { Disc herniation (in } 6 / 10 \text { patients) } \\
\text { Tumor }(3 / 10) \text {, Tuberculosis }\end{array}$ & Laminectomy and/or discectomy & $\begin{array}{l}\text { Leg pain and motor weakness were } \\
\text { improved in all patients after operation }\end{array}$ \\
\hline Kim et al. [45], 2007, CR, $n=1$ & Disc herniation & Discectomy & $\begin{array}{l}\text { Complete improvement of the pain but } \\
\text { partial improvement of neurological } \\
\text { deficits }\end{array}$ \\
\hline LaBan et al. [46], 1995, CS-R, $n=6$ & $\begin{array}{l}\text { Disc herniation (MRI - } 6 \text { patients) } \\
\text { Radiculopathy (EMG - } 4 \text { patients) }\end{array}$ & $\begin{array}{l}5 \text { cases - conservative } \\
1 \text { case - laminectomy }\end{array}$ & Not documented \\
\hline
\end{tabular}


Table 2d. (continued)

\begin{tabular}{|c|c|c|c|}
\hline Study author, year, type, number of patients & Finding on investigation & Treatment strategy & Outcome \\
\hline Lee et al. [18], 2011, CR, $n=1$ & Disc herniation & $\begin{array}{l}\text { Discectomy (intrapartum, left lateral } \\
\text { decubitus) }\end{array}$ & $\begin{array}{l}\text { Complete resolution of the pain and } \\
\text { neurological deficits }\end{array}$ \\
\hline \multicolumn{4}{|l|}{ Vertebral pathology } \\
\hline Bayram et al. [47], 2006, CR, $n=1$ & Vertebral fracture (osteoporotic) & Kyphoplasty & Resolution of pain \\
\hline Hsieh et al. [50], 2004, CR, $n=1$ & Vertebral fracture (spinal tumor) & $\begin{array}{l}\text { Surgical decompression and removal } \\
\text { of tumor }\end{array}$ & $\begin{array}{l}\text { Gradual and partial recovery of } \\
\text { neuro-deficit }\end{array}$ \\
\hline Kim et al. [48], 2010, CR, $n=1$ & Vertebral fractures (osteoporotic) & Percutaneous vertebroplasty & Resolution of pain \\
\hline $\begin{array}{l}\text { Moles et al. [51], 2014, ROL, } n=27 \\
(2+25)\end{array}$ & Vertebral hemangioma & Laminectomy & $\begin{array}{l}\text { Complete resolution of the pain and } \\
\text { neurological deficits }\end{array}$ \\
\hline \multicolumn{4}{|l|}{ Pelvic Girdle Pain } \\
\hline Desmond et al. [89], 2010, LTE, $n=1$ & Symphysis pubis pain & Symphysic pubis injection & Improvement in pain \\
\hline Hasegawa et al. [65], 1999, CR, $n=1$ & Posterior pelvic girdle pain & SIJ injection & Improvement in pain \\
\hline Hurdle et al. [90], 2007, CP, $n=4$ & Posterior pelvic girdle pain & SIJ injection & Improvement in pain \\
\hline
\end{tabular}

R, case report; CS-R, case series - retrospective; CO, cohort study; RCT, randomized controlled trial; NRCT, non-randomized controlled trial; LTE, letter to the editor; CP, conference proceeding; ROL, review of literature. Clinical syndromes: LBP, low back pain; PGP, pelvic girdle pain. Pain scores: VAS, Visual Analogue Scale; NRS, Numerical Rating Scale. Treatments: OMT, osteopathic manipulative treatment; ESI, epidural steroid injection. Investigations: MRI, magnetic resonance imaging; EMG, electromyography. Others: SIJ, sacroiliac joint.

nancy (20 vs. 26 weeks) or its intensity (superficial versus deep) did not alter its benefit for LBP [86, 87]. One study compared no treatment (control arm) to 3 intervention groups (exercise, acetaminophen and TENS respectively) and found TENS to be as effective as exercise and acetaminophen [88].

\section{Non-Surgical Interventions}

Three case reports described the injection of steroids into the epidural space (ESI) to relieve LBP and radicular pain [40-42]; but all patients eventually required operative intervention due to the recurrence or progression of neurological symptoms (Table 2d). Ultrasound-guided injection of local anaesthetic and steroid into the symphysis pubis joint in a pregnant patient to relieve severe symphysis pubis pain was also reported [89]. There were 2 reports of injections into the SIJ in pregnancy with good analgesic response $[65,90]$.

\section{Surgical Interventions}

Eight case reports and 2 case series described surgical interventions for intervertebral disc herniation causing neurological deficits (sensory, motor, bladder, or bowel involvement) including discectomy (open [38, 39, 41], micro-discectomy [18, 42], or endoscopic [45]), laminectomy [46], or both $[40,43,44]$ (Table 2d). Three case reports $[47,48,50]$ and one literature review [91] described surgical management of vertebral fractures and vertebral hemangiomas respectively. Surgery was generally successful in relieving pain and restoring function without increased morbidity or mortality.

\section{c. Use of Neuraxial Anaesthesia for Pregnant \\ Patients with LBP, Spinal Deformities, or Previous Spinal Surgery}

Two case series and 2 literature review described the use of neuraxial techniques (epidural or spinal injections and/or catheters) for labour analgesia or caesarean section in pregnant patients with a history of LBP (with/ without neurological deficits), spinal deformities and previous spinal surgery or implants (such as SCS; Table 3) $[17,92,93]$. While 2 publications focussed on the efficacy of interventions and technical feasibility $[92,93]$, one study also studied long-term neurological outcomes [17]. Although the techniques were successful in a majority of cases, there were multiple reports of complicated spinal or epidural injections (multiple insertion attempts, bloody taps and dural taps) $[92,93]$. There were also some technical (inability to place epidural catheters) and therapeutic (patchy blocks with missed dermatomes) failures.

Hebl et al. [17] retrospectively reviewed the risk of neurologic complications in 937 patients with a history of preexisting spinal canal pathology, with or without a history of spine surgery. This included 34 cases of labour analge- 
Table 3. Use of neuraxial analgesic or anesthetic techniques for pregnant patients with neurological deficits or previous spinal surgery

\begin{tabular}{|c|c|c|c|}
\hline $\begin{array}{l}\text { Study author, year, type, number of } \\
\text { patients }\end{array}$ & Population studied & Intervention & Outcomes \\
\hline Hebl et al. [17], 2010, CS-R, $n=937$ & $\begin{array}{l}\text { Previous spinal surgery lumbar radiculopathy } \\
\text { spinal stenosis other neuropathies included } \\
34 \text { cases of labor analgesia and } 10 \text { cases of } \\
\text { cesarian delivery }\end{array}$ & $\begin{array}{l}\text { Neuraxial anesthesia (spinal/epidural/combined) } \\
\text { for labor analgesia or cesarian section }\end{array}$ & $\begin{array}{l}10(1.1 \%) \text { cases of new } \\
\text { neurological or worsening of } \\
\text { pre-existing deficits in all comers } \\
\text { (pregnant and non-pregnant) }\end{array}$ \\
\hline Ko et al. [92], 2009, ROL, $n=103$ & $\begin{array}{l}93 \text { partituent with corrected scoliosis for labor } \\
\text { or operative analgesia }\end{array}$ & $\begin{array}{l}\text { Neuraxial anesthesia } \\
\text { (spinal/continuous spinal/ epidural/combined) } \\
\text { or others }\end{array}$ & $\begin{array}{l}64 \text { successful neuraxial } \\
\text { anesthetics, with } 2 \text { complications } \\
\text { (back pain post procedure) }\end{array}$ \\
\hline $\begin{array}{l}\text { Villevieille et al. [93], 2003, CS-R, } \\
n=20\end{array}$ & 20 patients with previous spinal surgery & $\begin{array}{l}\text { Epidural analgesia for labor or operative } \\
\text { anesthesia }\end{array}$ & $\begin{array}{l}2 \text { technical failures, } 2 \text { analgesic } \\
\text { failures }\end{array}$ \\
\hline $\begin{array}{l}\text { Young et al. [94], 2015, ROL, } \\
n=24\end{array}$ & $\begin{array}{l}\text { Authors reviewed neuraxial analgesia in } 17 \\
\text { cases with cervical/thoracic spinal cord } \\
\text { stimulator implants; authors described } \\
5 \text { cases ( } 4 \text { epidurals, } 1 \text { spinal); authors reviewed } \\
\text { literature ( } 5 \text { epidural, } 5 \text { spinal) }\end{array}$ & $\begin{array}{l}\text { Neuraxial anesthesia (spinal or epidural) } \\
\text { for labor or operative anesthesia }\end{array}$ & $\begin{array}{l}16 / 17 \text { attempts were successful. } \\
\text { One converted to GA } \\
1 \text { case of foot drop post-partum } \\
\text { (received epidural; unclear } \\
\text { etiology) }\end{array}$ \\
\hline
\end{tabular}

CS-R, case series - retrospective; ROL, review of literature.

sia and 10 caesarean sections. The incidence of new neurological deficits or worsening of pre-existing symptoms was $1.1 \%$ ( 10 out of 937 patients), though this study included both pregnant and non-pregnant populations. Further, data was not reported separately for the pregnant cohort.

Young et al. [94] retrospectively reviewed 24 cases of partituents with cervical or thoracic level SCS. Of the 17 cases in which a neuraxial was attempted for either labour analgesia or operative anaesthesia, 16 cases were successful. One patient who received epidural anaesthesia developed foot drop in the post-partum period. The etiology of this was unclear but assumed to be related to prolonged second stage of labor.

\section{Discussion}

Several physiological factors may contribute to low back and pelvic pain in pregnancy. This includes a rise in hormones (of relaxin, progesterone, and estrogen) possibly resulting in an increase in joint laxity [95-97]. Asymmetric SIJ laxity [66] and greater pubic symphysis mobility [98] are associated with moderate to severe posterior pelvic pain in pregnancy and puerperium. Structural imbalances created by weakened abdominal muscles (due to enlarged gravid uterus), compensatory hyperlordosis (sagittal rotation of pelvis) and shifting of centre of gravity anterior increase the load on lumbar spine and SIJ, contributing to back pain [97]. Axial loading of the spine may compress intervertebral discs, reduce

LBP in Pregnancy: Role of Neuraxial

Analgesia and Anaesthesia disc height and cause disc protrusion culminating in radicular symptoms. Osteoporosis in pregnancy has been associated with vertebral [48] and sacral stress fractures [60]. Other rare pathologies may include osteomyelitis [99] and spinal tumours [91, 100]. A detailed account of the aetiology of low back and pelvic pain in pregnancy can be found in other publications $[11,12]$.

This systematic review was performed to identify clinically useful and safe diagnostic investigations and management strategies for LBP in pregnancy and to make recommendations for neuraxial analgesia and anaesthesia during labour and delivery. The findings have been discussed below and recommendations outlined in Figure 3.

\section{Diagnostic Investigations}

The aetiological diagnosis of LBP is mostly clinical with imaging reserved for severe or challenging cases. Most common pathologies diagnosed on imaging in pregnancy include intervertebral disc herniations, vertebral hemangiomas and fractures and sacral stress fractures. MRI is the most commonly used imaging modality for pregnant women with neurological compromise (dermatomal lower limb numbness [38], perineal numbness $[39,40]$, lower limb weakness $[18,39,40,43,45,46]$, or loss of bladder/bowel control $[40,43,45])$. Suspected disc herniation was the most frequent reason for imaging, followed by incapacitating LBP without neurological compromise [42]. Unlike most patients with LBP and radicular lower limb pain that present in the second and the third trimesters, disc herniation was also reported in the first trimester [46]. This has implications for both diag-

Gynecol Obstet Invest 2017;82:417-436 DOI: $10.1159 / 000471764$ 
Fig. 3. Recommended algorithm for evaluation and management of LBP and PGP in pregnancy (interventional radiology includes modalities such as kyphoplasty and vertebroplasty, surgery includes laminectomy and discectomy).

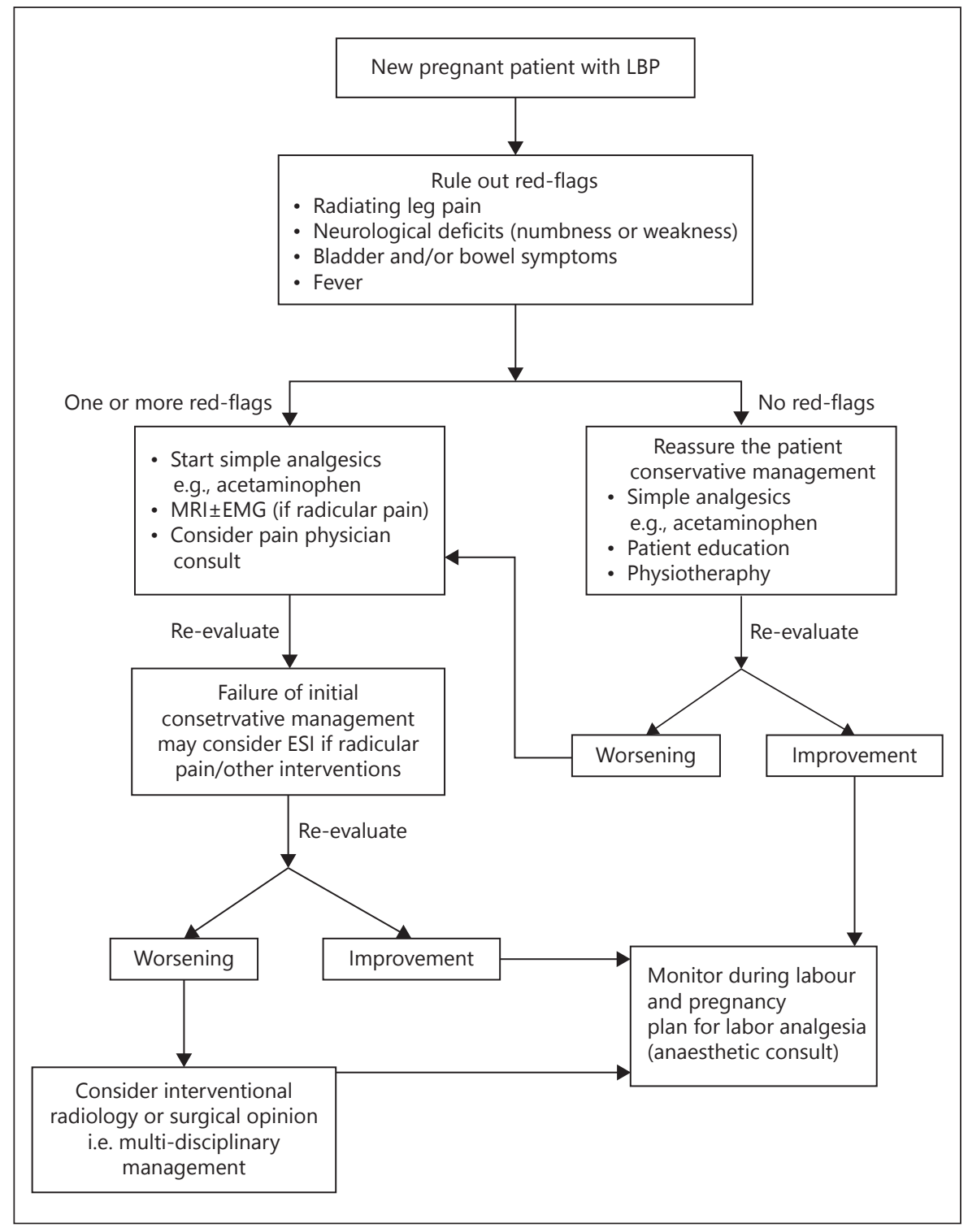

nostic imaging and interventions that are often delayed until after the first trimester.

MRI without contrast is considered the safest imaging modality for spinal pathologies that manifest during pregnancy $[11,101,102]$, offering better resolution when compared to ionizing imaging modalities (fluoroscopy and CT). While there have been concerns regarding MRI-induced fetal teratogenicity, acoustic damage and heating-induced effects, recent evidence does not support this $[103,104]$. However, the safety of the more recently introduced 3 Tesla MRI (which may provide better imaging because of a stronger magnetic field than the conventional 1.5 tesla MRI) has not yet been well studied [105].
There have been conflicting recommendations regarding the use of MRI in pregnancy, with some suggesting delaying its use until after the first trimester [106], while others proposing its use regardless of gestational age $[107,108]$. Since the intravenous contrast agent, gadolinium, could have teratogenic effects [109], caution in its use in pregnancy is recommended [108], although it is highly improbable that toxic levels would be attained in fetal circulation [110, 111]. Discarding breast milk for $24 \mathrm{~h}$ after its administration can minimize the less-intensive risk to the breast-fed infant.

The use of modalities with ionizing radiation (fluoroscopy and CT) for imaging in pregnancy has been associ- 
Table 4. Pain generator in lower back and pelvis, and summary of diagnostic investigations

\begin{tabular}{|c|c|c|c|}
\hline Pain generator & Presentation & Diagnostic modality of choice & Most common findings on imaging \\
\hline Intervertebral disc & $\begin{array}{l}\text { Disc protrusion or herniation causing radicular } \\
\text { symptoms such as pain, numbness, weakness } \\
\text { or loss of bowel/bladder control }\end{array}$ & $\begin{array}{l}\text { MRI scan }>\text { CT scan } \\
\text { EMG or NCS studies }\end{array}$ & $\begin{array}{l}\text { Herniation of disc or its contents posteriorly or } \\
\text { laterally, compressing the emerging spinal roots. } \\
\text { Altered nerve conduction study (delayed latency } \\
\text { and reduced amplitude or velocity of conduction) }\end{array}$ \\
\hline $\begin{array}{l}\text { Vertebral/sacral stress fracture or } \\
\text { collapse }\end{array}$ & $\begin{array}{l}\text { Mid or lower back pain without radicular } \\
\text { symptoms }\end{array}$ & CT scan $>$ MRI scan & $\begin{array}{l}\text { Fracture line or shear in vertebral or sacral body } \\
\text { (CT, MRI) } \\
\text { Peri fracture enhancement (osseous edema) due to } \\
\text { inflammation (MRI) }\end{array}$ \\
\hline SIJ laxity & Lower back pain and/or pelvic pain & Ultrasound & DIV to objectively measure the laxity of the SIJ \\
\hline Pubic diastasis & Anterior pelvic pain & Ultrasound, MRI & $\begin{array}{l}\text { Obvious gap between the pubic ramus } \\
\text { MRI may reveal focal edematous changes to the } \\
\text { para symphyseal pubis bones }\end{array}$ \\
\hline Pelvic floor muscles & Low back and/or pelvic pain & Ultrasound & $\begin{array}{l}\text { Pelvic floor muscles dysfunction: displacement of } \\
\text { pelvic floor elevation from resting position }\end{array}$ \\
\hline
\end{tabular}

SIJ, sacroiliac joint; DIV, doppler imaging of vibrations.

ated with the risk of spontaneous abortions, intrauterine growth restriction, neurobehavioral abnormalities, fetal malformations, or carcinogenesis [112, 113]. Such effects may depend upon the radiation dose absorbed by the fetus and the trimester of the pregnancy [114]. Although the estimated fetal absorption of radiation for a CT-scan of pelvis (20-79 $\mathrm{mGy}$ ) is below the significant risk threshold (100 mGy) [115], a dose of 100-200 mGy can be lethal for an embryo. Despite this, the fetal risks with clinically used doses are minimal and thus radiologic examinations that may provide significant diagnostic information should not be withheld from pregnant women [115]. Our review showed that CT scans were reserved for patients with upper back pain [116] or post-partum PGP [57, 58, $65]$, especially when thoracic vertebral collapse or associated pathology in the hip was suspected. The reason for this is that CT is often preferred over MRI when bony pathology is suspected [117].

Although ultrasound is considered safe in pregnancy, it does not provide the information required to evaluate most spinal pathologies [118]. The indications for using diagnostic ultrasound in patients with LBP include suspected SIJ pathology [20, 119], diagnosing and monitoring pubic diastasis, and the assessment of pelvic floor dysfunction [120]. Clinical evaluation and MRI may not accurately diagnose SIJ dysfunction.

Electro-diagnostic studies (NCS and EMG) are valuable tools to supplement anatomical information obtained from imaging in pregnant women with lumbar radiculopathy secondary to nerve root compression by intervertebral disc disease, ligamentous hypertrophy or arthritic changes [121-123]. These can help ascertain the correlation between MRI findings and presenting neurologic deficits, allowing an informed decision to perform a therapeutic injection or operative intervention [124]. Despite this, we found a low rate of use for electrodiagnostic studies, which may be due to patient preference (electrodiagnostic studies can be painful), urgency of the presentation or lack of awareness among health care providers.

Table 4 summarized the common pain generators in the lower back and pelvis, their common presentations, imaging or investigations of choice and their common findings. It must be borne in mind that the correlation between the causes of back pain and the radiological findings is poor $[125,126]$ and that findings on imaging do not always imply aetiology [127].

\section{Therapies for Treatment/Management of}

\section{LBP in Pregnancy}

Multimodal management of LBP that includes physical therapy, patient education and psychological therapies, complimentary treatments, and interventional techniques (injections and surgery) is recommended for optimizing outcomes in non-pregnant patients [128]. The role of these modes of therapy in pregnant women with LBP is discussed below.

\section{Multimodal Management Including Patient}

Education, Exercise, and Psychological Therapies

Back care training programs educate pregnant women on the basic anatomy, ergonomics, correct postures, relaxation and pain management strategies [11]. Although our review shows conflicting evidence of their role, it promotes awareness on the benefits of being active and of 
self-care, potentially reducing the severity of LBP and consequent disability. The National Institute for Health and Clinical Excellence in the United Kingdom (UK) recommends that "exercising in water, massage therapy and group or individual back care classes" may help in reducing LBP during pregnancy [129]. Bio-psychosocial factors (such as anxiety and catastrophisation) can prevent the conditioning of back muscles and worsening LBP [58, $120]$, both during and after pregnancy [131], and should be considered when formulating preventive strategies and in rehabilitation.

\section{Physical Therapy and Exercise}

The evidence for exercise in management of LBP in pregnancy is equivocal. The results of 2 recent systematic reviews differ from each other, probably due to the inclusion of post-partum women in one [132] and not in the other [133]. Although exercise does not appear to benefit patients with PGP $[23,32,33]$, it is often recommended during pregnancy because it is a non-invasive, low-risk, inexpensive intervention that may have benefits in addition to providing relief from back pain $[129,134]$.

\section{Complementary and Alternative Medicine}

Pelvic belts and other support devices may limit the laxity at SIJ, provide symptomatic relief, and are safe [135]. However, their use is not supported in a recent systemic review of lumbar supports [136]. While there is a paucity of RCTs utilizing yoga for alleviating LBP in pregnancy, meta-analysis from non-pregnant populations with LBP found moderate-to-strong evidence of its effectiveness [137] with the caveats that pain-related catastrophising and fear of movement may decrease its use [138, 139]. Though 2 RCTs support the use of acupuncture for managing LBP and PGP in pregnancy $[22,36]$, evidence for acupuncture in managing LBP is uncertain, mainly due to methodological flaws and significant heterogeneity among included studies [140-142]. Based on one RCT, TENS appears to be as effective as exercise therapy or acetaminophen at reducing both pain and disability from pregnancy-related back pain.

\section{Non-Surgical Interventions}

We found weak evidence for the analgesic and surgery-delaying effect of ESI in pregnant patients with LBP, which is consistent with observations in non-pregnant patients $[128,143]$. Although a single dose of epidural steroid appears to be of low risk to the fetus, it is recommended that ESI be reserved for patients with new onset of signs or severe symptoms of lumbar nerve root compression [14]. Unknown variables about ESI in pregnant patients include frequency of its use, efficacy, and duration of therapeutic effect. It might, however, be reasonable to use higher volume (5-10 mL) ESI based on clinical diagnosis, whereas recurrence or worsening of neurological symptoms may prompt imaging and a transforaminal ESI using lower volumes $(1.5-3 \mathrm{~mL})$ that is performed with the objective of the medication reaching the dorsal root ganglion of the spinal nerve root [144].

\section{Surgical Interventions}

Lumbar spine surgery under general anaesthesia [3841 ] is indicated during pregnancy for disc herniation, tumours and vertebral fractures associated with significant neurological compromise. Although surgery on the lumbosacral spine in pregnant women poses hazards to the mother and fetus because of requirement for prone or lateral positioning and exposure to anaesthetic medications, it should not be delayed if essential for preserving maternal life and function [145]. Preoperative discussion regarding risks with the pregnant patient and a team approach involving obstetricians, anaesthesiologists, neonatologists and spine surgeons is advocated for optimizing outcomes [44]. Co-interventions include perioperative fetal monitoring after 23 weeks of gestation [41], administration of corticosteroids to accelerate fetal lung maturity before 34 weeks of gestation and fetal surveillance in the immediate postoperative period [146]. Delivery is rarely indicated to allow spinal surgery at the same time $[38,39,41,43]$. Prone position may be utilized to access the spine during the first trimester or in post-partum $[39,41]$, while lateral decubitus that reduces aortocaval compression, is preferable in second and third trimesters [45].

\section{Use of Neuraxial Anaesthesia for Pregnant}

Patients with LBP, Spinal Deformities, or

Previous Spinal Surgery

We found that neuraxial techniques (epidural or spinal) in pregnant patients with history of LBP, spinal deformities or previous spinal surgery are associated with higher rates of complications and failures. It is noteworthy that the risk of neurological complications reported by Hebl et al. [17] is considerably higher than that found as a result of a UK national audit (1.1/100 vs. $2.0-4.2 / 100,000)$, although the evidence synthesized by Hebl et al. [17] included both pregnant and nonpregnant patients [147]. These findings may lead to anaesthesiologists not offering neuraxial techniques to 
pregnant patients with LBP because of the potential for worsening of pre-existing neuraxial deficits. This is unfortunate because neuraxial analgesia and anaesthesia have a better efficacy and safety profile than systemic analgesia and general anaesthesia $[148,149]$. Although selecting a different level than the level of pathology to perform the neuraxial techniques may appear reasonable, there is not much evidence to support this theory. In their review, Hebl et al. [17] found that 7 out of 10 patients who reported new or worsening neurological deficits had a neuraxial block at a different level than the level of the pathology. These results cannot be confidently interpreted because the palpatory method of spinal level localization utilized in this review is unreliable [150]. Despite this, avoiding the level of an obvious or known pathology by use of an ultrasound-guided technique may be considered potentially protective until further evidence is available. The use of ultrasound for guiding neuraxial procedures helps in better identification of spinal levels, allows accurate estimation of midline and epidural depth, improves success rate and ease of performance and may reduce the risk of traumatic procedures [151]; this is applicable to patients with previous spine surgery [152]. We envision expansion in the use of ultrasound-guided neuraxial interventions in parturients and further research is in progress [153].

Technical feasibility and efficacy of neuraxial anaesthesia particularly after spinal surgery are other valid concerns for healthcare providers. Epidural anaesthesia can be challenging in such patients due to abnormal or missing landmarks, and due to the presence of surgical implants. It is associated with a higher risk of unintentional dural puncture, false loss of resistance, multiple puncture attempts and consequent patchy or failed blocks [92, 93, 154-156]. In patients with previous spine surgery, a "single-shot" spinal anaesthetic may be a more reliable and efficacious approach [17]. Combined spinal-epidural [157] and continuous spinal anaesthesia [158] have also been successfully utilized in patients with previous spinal surgery but are less common choices due to perceived technical difficulty. Thankfully, the presence of spinal implants does not preclude the possibility or impact the success of neuraxial anaesthesia or analgesia in patients with a spinal cord stimulator [94]. This is because most such patients have implants at either cervical or lower thoracic levels. However, necessary details pertaining to this level, and preferably implant-related spine imaging, must be reviewed to avoid inadvertent damage to the epidurally placed leads.

LBP in Pregnancy: Role of Neuraxial

Analgesia and Anaesthesia
Timely identification and planning of care by a multidisciplinary team for patients with severe LBP, spine pathology, or history of spinal surgery or implants in the antenatal clinic can assist appropriate planning of labour analgesia or surgical management for delivery. It is also important to note that delivery itself can cause neurological damage resulting in pain and disability post-partum [159]. Additionally, there are concerns that repeated Valsalva manoeuvers for vaginal delivery may further compress the cauda equina in a patient with disc herniation and cause worsening of neurological deficit [41]. Therefore, in cases with pre-existing neurological deficits, decisions regarding the management of labour and delivery must be made by a multidisciplinary team. The use of neuraxial anaesthesia, especially labour analgesia, may also potentially prevent the prompt recognition of worsening neurological deficits. Finally, while it is not anticipated that the risk for neurological injury in patients with LBP risk may be increased, a documentation of the patient's LBP may help differentiate this from other causes of post-partum back pain.

\section{Strengths and Limitations of this Systematic Review}

This systematic review employed a comprehensive search strategy covering all aspects in the management of LBP in pregnancy with evidence-based recommendations. However, due to the absence of high-level evidence in all areas, a significant proportion of evidence came from cohort studies, case series and case reports, and this increases the possibility of bias. The RCTs included in this review were of moderate quality and included several single blind studies that may influence the outcomes, particularly in interventional studies. Evidence regarding the use of neuraxial analgesia and anaesthesia is largely from non-pregnant patients. Unique anatomical and physiological changes of pregnancy suggest that inferences based on studies on non-pregnant patients may have limited applicability in pregnancy.

\section{Conclusions}

LBP is common in pregnancy, but there are several knowledge gaps regarding optimal modalities for diagnosis, treatment, and anaesthetic management of this condition. The evidence presented in this review suggests that though sinister causes of LBP are uncommon, "red-flags" such as radiating leg pain, neurologi-

Gynecol Obstet Invest 2017;82:417-436

DOI: $10.1159 / 000471764$ 
cal deficits (numbness or weakness), bladder and/or bowel symptoms, or fever should be excluded in all patients. While the evaluation of LBP is mostly clinical, MRI is the diagnostic investigation of choice during pregnancy, with recourse to imaging involving ionizing radiation, ultrasound and electro-diagnostic studies in some instances. The inclusion of back care training in antenatal educational programs that include consideration of bio-psychosocial factors may promote activity and help reduce morbidity in pregnancy. There is limited evidence for the role of exercise and acupuncture and further research is needed in establishing the role of complementary and alternative therapies in the management of LBP in pregnancy. ESI for pregnant patients with radicular pain may confer analgesic benefit, while operative intervention is indicated for the most severe cases of LBP, particularly with associated neurological compromise (Fig. 3). Performance of neuraxial blocks in pregnant patients with LBP, neurological deficits and previous spine surgery or implants is technically feasible in most cases, but until more evidence is available regarding neurological complications in pregnant patients with neurological deficits or previous spinal surgery, management should be individualised.

\section{Acknowledgements}

We would like to thank Ms. Marina Engelasakis, Librarian, University Health Network for her assistance in designing the literature search strategy.

\section{Disclosure Statement}

The authors have no conflicts of interests to disclose.

\section{Contribution to Authorship}

A.B. and H.S. designed the study, reviewed the literature, analysed the data, and prepared the manuscript. R.D. provided obstetrical and methodological input and helped with editing the manuscript.

\section{Ethics Approval}

Not applicable.

\section{Funding}

The authors have no funding to declare.

\section{References}

1 Fast A, Shapiro D, Ducommun EJ, Friedmann LW, Bouklas T, Floman Y: Low-back pain in pregnancy. Spine (Phila Pa 1976) 1987;12: 368-371.

2 Ostgaard HC, Andersson GB, Karlsson K: Prevalence of back pain in pregnancy. Spine (Phila Pa 1976) 1991;16:549-552.

3 Wu WH, Meijer OG, Uegaki K, Mens JM, van Dieen JH, Wuisman PI, et al: Pregnancy-related pelvic girdle pain (PPP), I: terminology, clinical presentation, and prevalence. Eur Spine J 2004; 13:575-589.

4 Ostgaard HC, Andersson GB: Previous back pain and risk of developing back pain in a future pregnancy. Spine (Phila Pa 1976) 1991; 16:432-436.

5 Albert H, Godskesen M, Westergaard J: Prognosis in four syndromes of pregnancy-related pelvic pain. Acta Obstet Gynecol Scand 2001; 80:505-510.

6 Rost CC, Jacqueline J, Kaiser A, Verhagen AP, Koes BW: Prognosis of women with pelvic pain during pregnancy: a long-term followup study. Acta Obstet Gynecol Scand 2006;85: 771-777.

7 Ostgaard HC, Andersson GB, Wennergren $\mathrm{M}$ : The impact of low back and pelvic pain in pregnancy on the pregnancy out- come. Acta Obstet Gynecol Scand 1991;70: 21-24.

8 Aune D, Saugstad OD, Henriksen T, Tonstad $\mathrm{S}$ : Physical activity and the risk of preeclampsia: a systematic review and meta-analysis. Epidemiology 2014;25:331-343.

9 Domenjoz I, Kayser B, Boulvain M: Effect of physical activity during pregnancy on mode of delivery. Am J Obstet Gynecol 2014;211: 401.e1-e11.

10 Price BB, Amini SB, Kappeler K: Exercise in pregnancy: effect on fitness and obstetric outcomes - a randomized trial. Med Sci Sports Exerc 2012;44:2263-2269.

11 Vermani E, Mittal R, Weeks A: Pelvic girdle pain and low back pain in pregnancy: a review. Pain Pract 2010;10:60-71.

12 Casagrande D, Gugala Z, Clark SM, Lindsey RW: Low back pain and pelvic girdle pain in pregnancy. J Am Acad Orthop Surg 2015;23: 539-549.

13 Black RA, Hill DA: Over-the-counter medications in pregnancy. Am Fam Physician 2003; 67:2517-2524.

14 Rathmell JP, Viscomi CM, Ashburn MA: Management of nonobstetric pain during pregnancy and lactation. Anesth Analg 1997; 85:1074-1087.
15 American Academy of Pediatrics Committee on Drugs: Transfer of drugs and other chemicals into human milk. Pediatrics 2001;108: 776-789.

16 Vercauteren M, Waets P, Pitkanen M, Forster $\mathrm{J}$ : Neuraxial techniques in patients with preexisting back impairment or prior spine interventions: a topical review with special reference to obstetrics. Acta Anaesthesiol Scand 2011;55:910-917.

17 HeblJR, HorlockerTT,KoppSL,Schroeder DR: Neuraxial blockade in patients with preexisting spinal stenosis, lumbar disk disease, or prior spine surgery: efficacy and neurologic complications. Anesth Analg 2010;111:1511-1519.

18 Lee JM, Han IH, Moon SH, Choi BK: Surgery for recurrent lumbar disc herniation during pregnancy: a case report. Korean J Spine 2011; 8:304-306.

19 Moher D, Liberati A, Tetzlaff J, Altman DG; PRISMA Group: Preferred reporting items for systematic reviews and meta-analyses: the PRISMA statement. Ann Intern Med 2009; 151:264-269.

20 Vleeming A, Albert HB, Östgaard HC, Sturesson B, Stuge B: European guidelines for the diagnosis and treatment of pelvic girdle pain. Eur Spine J 2008;17:794-819. 
21 Higgins JP, Altman DG, Gotzsche PC, Juni P, Moher D, Oxman AD, et al: The Cochrane Collaboration's tool for assessing risk of bias in randomised trials. BMJ 2011;343:d5928.

22 Guerreiro da Silva JB, Nakamura MU, Cordeiro JA, Kulay L Jr: Acupuncture for low back pain in pregnancy - a prospective, quasirandomised, controlled study. Acupunct Med 2004;22:60-67.

23 Nilsson-Wikmar L, Holm K, Oijerstedt R, Harms-Ringdahl K: Effect of three different physical therapy treatments on pain and activity in pregnant women with pelvic girdle pain: a randomized clinical trial with 3,6 , and 12 months follow-up postpartum. Spine (Phila Pa 1976) 2005;30:850-856.

24 Ostgaard HC, Zetherstrom G, Roos-Hansson E, Svanberg B: Reduction of back and posterior pelvic pain in pregnancy. Spine (Phila $\mathrm{Pa}$ 1976) 1994;19:894-900.

25 Ostgaard HC, Zetherstrom G, Roos-Hansson E: Back pain in relation to pregnancy: a 6-year follow-up. Spine (Phila Pa 1976) 1997;22: 2945-2950.

26 Licciardone JC, Buchanan S, Hensel KL, King HH, Fulda KG, Stoll ST: Osteopathic manipulative treatment of back pain and related symptoms during pregnancy: a randomized controlled trial. Am J Obstet Gynecol 2010; 202:43.e1-e8.

27 Eggen MH, Stuge B, Mowinckel P, Jensen KS, Hagen KB: Can supervised group exercises including ergonomic advice reduce the prevalence and severity of low back pain and pelvic girdle pain in pregnancy? A randomized controlled trial. Phys Ther 2012;92:781-790.

28 Mens JM, Snijders CJ, Stam HJ: Diagonal trunk muscle exercises in peripartum pelvic pain: a randomized clinical trial. Phys Ther 2000;80:1164-1173.

29 Stuge B, Laerum E, Kirkesola G, Vøllestad N: The efficacy of a treatment program focusing on specific stabilizing exercises for pelvic girdle pain after pregnancy: a randomized controlled trial. Spine (Phila Pa 1976) 2004;29: 351-359.

30 Akmese ZB, Oran NT: Effects of progressive muscle relaxation exercises accompanied by music on low back pain and quality of life during pregnancy. J Midwifery Womens Health 2014;59:503-509.

31 Bastiaenen CH, Bastiaanssen JM, de Bie RA Re: Gutke A, Ostgaard HC, Oberg B: Pelvic girdle pain and lumbar pain in pregnancy: a cohort study of the consequences in terms of health and functioning. Spine 2006;31:E14955. Spine (Phila Pa 1976) 2006;31:2406; author reply 2406-2407.

32 Elden H: Effects of acupuncture and stabilising exercises as adjunct to standard treatment in pregnant women with pelvic girdle pain: randomised single blind controlled trial. BMJ 2005;330:761.

33 Elden H, Fagevik-Olsen M, Ostgaard HC, Stener-Victorin E, Hagberg H: Acupuncture as an adjunct to standard treatment for pelvic girdle pain in pregnant women: ran- domised double-blinded controlled trial comparing acupuncture with non-penetrating sham acupuncture. BJOG 2008;115: $1655-1668$.

34 Gutke A, Sjodahl J, Oberg B: Specific muscle stabilizing as home exercises for persistent pelvic girdle pain after pregnancy: a randomized, controlled clinical trial. J Rehabil Med 2010;42:929-935.

35 Kihlstrand M, Stenman B, Nilsson S, Axelsson O: Water-gymnastics reduced the intensity of back/low back pain in pregnant women. Acta Obstet Gynecol Scand 1999;78:180185.

36 Kvorning N, Holmberg C, Grennert L, Aberg A, Akeson J: Acupuncture relieves pelvic and low-back pain in late pregnancy. Acta Obstet Gynecol Scand $2004 ; 83: 246-250$.

37 Haugland KS, Rasmussen S, Daltveit AK: Group intervention for women with pelvic girdle pain in pregnancy. A randomized controlled trial. Acta Obstet Gynecol Scand 2006; 85:1320-1326.

38 Abou-Shameh MA, Dosani D, Gopal S, McLaren AG: Lumbar discectomy in pregnancy. Int J Gynaecol Obstet 2006;92:167169.

39 Al-areibi A, Coveney L, Singh S, Katsiris S: Case report: anesthetic management for sequential Cesarean delivery and laminectomy. Can J Anaesth 2007;54:471-474.

40 Brown MD, Levi AD: Surgery for lumbar disc herniation during pregnancy. Spine (Phila $\mathrm{Pa}$ 1976) 2001;26:440-443.

41 Brown MD, Brookfield KF: Lumbar disc excision and cesarean delivery during the same anesthesia. A case report. J Bone Joint Surg Am 2004;86:2030-2032.

42 Darwich AA, Diwan SA: Management of back pain in pregnancy. Tech Reg Anesth Pain Manag 2009;13:251-254.

43 Garmel SH, Guzelian GA, D'Alton JG, D'Alton ME: Lumbar disk disease in pregnancy. Obstet Gynecol 1997;89(5 pt 2):821-822.

44 Han IH, Kuh SU, Kim JH, Chin DK, Kim KS, Yoon YS, et al: Clinical approach and surgical strategy for spinal diseases in pregnant women: a report of ten cases. Spine (Phila Pa 1976) 2008;33:E614-E619.

45 Kim HS, Kim SW, Lee SM, Shin H: Endoscopic discectomy for the cauda equina syndrome during third trimester of pregnancy. J Korean Neurosurg Soc 2007;42:419-420.

46 LaBan MM, Rapp NS, von Oeyen P, Meerschaert JR: The lumbar herniated disk of pregnancy: a report of six cases identified by magnetic resonance imaging. Arch Phys Med Rehabil 1995;76:476-479.

47 Bayram S, Ozturk C, Sivrioglu K, Aydinli U, Kucukoglu S: Kyphoplasty for pregnancy-associated osteoporotic vertebral fractures. Joint Bone Spine 2006;73:564-566.

48 Kim HW, Song JW, Kwon A, Kim IH: Percutaneous vertebroplasty for pregnancy-associated osteoporotic vertebral compression fractures. J Korean Neurosurg Soc 2010;47:399402.
49 Ozdemir D, Tam AA, Dirikoc A, Ersoy R, Cakir B: Postpartum osteoporosis and vertebral fractures in two patients treated with enoxaparin during pregnancy. Osteoporos Int 2015; 26:415-418.

50 Hsieh HP, Po HL, Hseuh IH: Low back pain in a pregnant woman. Acta Neurol Taiwan 2004;13:84-86.

51 Moles A, Hamel O, Perret C, Bord E, Robert R, Buffenoir K: Symptomatic vertebral hemangiomas during pregnancy. J Neurosurg Spine 2014;20:585-591.

52 Egerman RS, Mabie WC, Eifrid M, Whitnack E, Sibai BM: Sacroiliitis associated with pyelonephritis in pregnancy. Obstet Gynecol 1995; 85:834-835.

53 Mulvey JM: Postpartum septic sacroiliitis coincident with labour epidural analgesia. Anaesth Intensive Care 2008;36:875-878.

54 Park YS, Owen AM, Adno AM, Marry J: Pyogenic sacroiliitis due to group A streptococcus following uncomplicated pregnancy and vaginal delivery. Case Rep Obstet Gynecol 2013; 2013:981474

55 Giannoulis DK, Koulouvaris P, Zilakou E, Papadopoulos DB, Lykissas MG, Mavrodontidis AN: Atraumatic sacral fracture in late pregnancy: a case report. Global Spine J 2015;5: 248-251.

56 Goeb V, Strotz V, Verdet M, Le Loet X, Vittecoq O: Post-partum sacral fracture associated with heparin treatment. Clin Rheumatol 2008;27(suppl 2):S51-S53.

57 Karatas M, Basaran C, Ozgul E, Tarhan C, Agildere AM: Postpartum sacral stress fracture: an unusual case of low-back and buttock pain. Am J Phys Med Rehabil 2008;87:418422.

58 Onur O, Atalar H, Cakirbay H, Gok K: Postpartum sacral stress fracture: case report. Turkiye Klin Jinekoloji Obstet 2011;21:42-46.

59 Ozturk G, Kulcu DG, Aydog E: Intrapartum sacral stress fracture due to pregnancy-related osteoporosis: a case report. Arch Osteoporos 2013;8:139.

60 Park J, Ok E, Park HJ, Hong SH, Lee JI: Postpartum sacral stress fracture mimicking lumbar radiculopathy in a patient with pregnancy-associated osteoporosis. Ann Rehabil Med 2013;37:582-585.

61 Pishnamaz M, Sellei R, Pfeifer R, Lichte P, Pape HC, Kobbe P: Low back pain during pregnancy caused by a sacral stress fracture: a case report. J Med Case Rep 2012;6:98.

62 Rousiere M, Kahan A, Job-Deslandre C: Postpartal sacral fracture without osteoporosis. Joint Bone Spine 2001;68:71-73.

63 Sansone V, McCleery J, Bonora C: Post-partum low-back pain of an uncommon origin: a case report. J Back Musculoskelet Rehabil 2013;26:475-477.

64 Thein R, Burstein G, Shabshin N: Labor-related sacral stress fracture presenting as lower limb radicular pain. Orthopedics 2009;32: 447.

65 Hasegawa Y, Iwata H: Chronic pelvic girdle relaxation. Jpn J Rheumatol 1999;9:391-395.
LBP in Pregnancy: Role of Neuraxial Analgesia and Anaesthesia
Gynecol Obstet Invest 2017;82:417-436 DOI: $10.1159 / 000471764$ 
66 Damen L, Buyruk HM, Guler-Uysal F, Lotgering FK, Snijders CJ, Stam HJ: Pelvic pain during pregnancy is associated with asymmetric laxity of the sacroiliac joints. Acta Obstet Gynecol Scand 2001;80:1019-1024.

67 Arab AM, Behbahani RB, Lorestani L, Azari A: Assessment of pelvic floor muscle function in women with and without low back pain using transabdominal ultrasound. Man Ther 2010;15:235-239.

68 Bastiaenen $\mathrm{CH}$, de Bie RA, Wolters PM, Vlaeyen JW, Leffers P, Stelma F, et al: Effectiveness of a tailor-made intervention for pregnancy-related pelvic girdle and/or low back pain after delivery: short-term results of a randomized clinical trial [ISRCTN08477490]. BMC Musculoskelet Disord 2006;7:19.

69 Mantle MJ, Holmes J, Currey HL: Backache in pregnancy II: prophylactic influence of back care classes. Rheumatol Rehabil 1981;20:227232.

70 George JW, Skaggs CD, Thompson PA, Nelson DM, Gavard JA, Gross GA: A randomized controlled trial comparing a multimodal intervention and standard obstetrics care for low back and pelvic pain in pregnancy. Am J Obstet Gynecol 2013;208:295.e1-e7.

71 Miquelutti MA, Cecatti JG, Makuch MY: Evaluation of a birth preparation program on lumbopelvic pain, urinary incontinence, anxiety and exercise: a randomized controlled trial. BMC Pregnancy Childbirth 2013;13: 154.

72 Garshasbi A, Faghih Zadeh S: The effect of exercise on the intensity of low back pain in pregnant women. Int J Gynecol Obstet 2005; 88:271-275.

73 Andrews CM, O’Neill LM: Use of pelvic tilt exercise for ligament pain relief. J Nurse Midwifery 1994;39:370-374.

74 Kluge J, Hall D, Louw Q, Theron G, Grové D: Specific exercises to treat pregnancy-related low back pain in a South African population. Int J Gynaecol Obstet 2011;113:187-191.

75 Dumas GA, Reid JG, Wolfe LA, Griffin MP, McGrath MJ: Exercise, posture, and back pain during pregnancy. Part 2. Exercise and back pain. Clin Biomech 1995;10:104-109.

76 Kashanian M, Akbari Z, Alizadeh MH: The effect of exercise on back pain and lordosis in pregnant women. Int J Gynaecol Obstet 2009; 107:160-161.

77 Haakstad LA, Bø K: Effect of a regular exercise programme on pelvic girdle and low back pain in previously inactive pregnant women: a randomized controlled trial. J Rehabil Med 2015;47:229-234.

78 Stafne SN, Salvesen KÅ, Romundstad PR, Stuge B, Mørkved S: Does regular exercise during pregnancy influence lumbopelvic pain? A randomized controlled trial. Acta Obstet Gynecol Scand 2012;91:552-559.

79 Mens JM, Damen L, Snijders CJ, Stam HJ: The mechanical effect of a pelvic belt in patients with pregnancy-related pelvic pain. Clin Biomech (Bristol, Avon) 2006;21:122-127.
80 Kalus S, Kornman LH, Quinlivan JA: Managing back pain in pregnancy using a support garment: a randomised trial. BJOG 2007;115: 68-75.

81 Hiemstra LA, Kerslake S, Irving C: Anterior knee pain in the athlete. Clin Sports Med 2014;33:437-459.

82 Schwerla F, Rother K, Rother D, Ruetz M, Resch KL: Osteopathic manipulative therapy in women with postpartum low back pain and disability: a pragmatic randomized controlled trial. J Am Osteopath Assoc 2015;115:416425.

83 Martins RF, Pinto e Silva JL: Treatment of pregnancy-related lumbar and pelvic girdle pain by the yoga method: a randomized controlled study. J Altern Complement Med 2014;20:24-31.

84 Cummings M: Acupuncture for low back pain in pregnancy. Acupunct Med 2003;21: $42-46$.

85 Forrester M: Low back pain in pregnancy. Acupunct Med 2003;21:36-41.

86 Ekdahl L, Petersson K; Acupuncture treatment of pregnant women with low back and pelvic pain - an intervention study. Scand J Caring Sci 2010;24:175-182.

87 Lund I, Lundeberg T, Lönnberg L, Svensson $\mathrm{E}$ : Decrease of pregnant women's pelvic pain after acupuncture: a randomized controlled single-blind study. Acta Obstet Gynecol Scand 2006;85:12-19.

88 Keskin EA, Onur O, Keskin HL, Gumus II, Kafali H, Turhan N: Transcutaneous electrical nerve stimulation improves low back pain during pregnancy. Gynecol Obstet Invest 2012;74:76-83.

89 Desmond FA, Harmon D: Ultrasound-guided symphysis pubis injection in pregnancy. Anesth Analg 2010;111:1329-1330.

90 Hurdle M-FB, McHugh R, Schwendemann W, Psimos C, Smith J: Poster 128: ultrasound guided sacroiliac joint injection in pregnancy: a case series. Arch Phys Med Rehabil 2007; 88:E45-E46.

91 Moles A, Hamel O, Perret C, Bord E, Robert R, Buffenoir K: Symptomatic vertebral hemangiomas during pregnancy. J Neurosurg Spine 2014;20:585-591.

92 Ko JY, Leffert LR: Clinical implications of neuraxial anesthesia in the parturient with scoliosis. Anesth Analg 2009;109:1930-1934.

93 Villevieille T, Mercier FJ, Benhamou D: [Is obstetric epidural anaesthesia technically possible after spinal surgery and does it work?]. Ann Fr Anesth Reanim 2003;22:9195.

94 Young AC, Lubenow TR, Buvanendran A: The parturient with implanted spinal cord stimulator: management and review of the literature. Reg Anesth Pain Med 2015;40:276283.

95 Aldabe D, Ribeiro DC, Milosavljevic S, Dawn Bussey M: Pregnancy-related pelvic girdle pain and its relationship with relaxin levels during pregnancy: a systematic review. Eur Spine J 2012;21:1769-1776.
96 Ireland ML, Ott SM: The effects of pregnancy on the musculoskeletal system. Clin Orthop Relat Res 2000;169-179.

97 Sneag DB, Bendo JA: Pregnancy-related low back pain. Orthopedics 2007;30:837-839.

98 Mens JM, Pool-Goudzwaard A, Stam HJ; Mobility of the pelvic joints in pregnancyrelated lumbopelvic pain: a systematic review. Obstet Gynecol Surv 2009;64:200-208.

99 Moros ML, Rodrigo C, Villacampa A, Ruiz J, Lapresta C: Septic shock in pregnancy due to pyogenic sacroiliitis: a case report. J Med Case Rep 2009;3:6505.

100 Han IH, Kuh SU, Kim JH, Chin DK, Kim KS, Yoon YS, et al: Clinical approach and surgical strategy for spinal diseases in pregnant women: a report of ten cases. Spine (Phila Pa 1976) 2008;33:E614-E619.

101 Sabino J, Grauer JN: Pregnancy and low back pain. Curr Rev Musculoskelet Med 2008;1:137-141.

102 Kanakaris NK, Roberts CS, Giannoudis PV: Pregnancy-related pelvic girdle pain: an update. BMC Med 2011;9:15.

103 Choi JS, Ahn HK, Han JY, Han YJ, Kwak DO, Velazquez-Armenta EY, et al: A case series of 15 women inadvertently exposed to magnetic resonance imaging in the first trimester of pregnancy. J Obstet Gynaecol 2015;35:871-872.

104 Bouyssi-Kobar M, du Plessis AJ, Robertson RL, Limperopoulos C: Fetal magnetic resonance imaging: exposure times and functional outcomes at preschool age. Pediatr Radiol 2015;45:1823-1830.

105 Victoria T, Jaramillo D, Roberts TP, Zarnow D, Johnson AM, Delgado J, et al: Fetal magnetic resonance imaging: jumping from 1.5 to 3 tesla (preliminary experience). Pediatr Radiol 2014;44:376-386; quiz 373-375.

106 Medical magnetic resonance (MR) procedures: protection of patients. Health Phys 2004;87:197-216.

107 ACOG Committee Opinion. Number 299, September 2004 (replaces No. 158, September 1995). Guidelines for diagnostic imaging during pregnancy. Obstet Gynecol 2004;104: 647-651.

108 Kanal E, Barkovich AJ, Bell C, Borgstede JP, Bradley WG Jr, Froelich JW, et al: ACR guidance document on MR safe practices: 2013. J Magn Reson Imaging 2013;37:501530.

109 Garcia-Bournissen F, Shrim A, Koren G: Safety of gadolinium during pregnancy. Can Fam Physician 2006;52:309-310.

110 Tremblay E, Thérasse E, Thomassin-Naggara I, Trop I: Quality initiatives: guidelines for use of medical imaging during pregnancy and lactation. Radiographics 2012;32:897911.

111 Wang PI, Chong ST, Kielar AZ, Kelly AM, Knoepp UD, Mazza MB, et al: Imaging of pregnant and lactating patients: part $1, \mathrm{ev}-$ idence-based review and recommendations. AJR Am J Roentgenol 2012;198:778884 . 
112 Masselli G, Derchi L, McHugo J, Rockall A, Vock P, Weston M, et al: Acute abdominal and pelvic pain in pregnancy: ESUR recommendations. Eur Radiol 2013;23:3485-3500.

113 Groen RS, Bae JY, Lim KJ: Fear of the unknown: ionizing radiation exposure during pregnancy. Am J Obstet Gynecol 2012;206: 456-462.

114 Brent RL: Saving lives and changing family histories: appropriate counseling of pregnant women and men and women of reproductive age, concerning the risk of diagnostic radiation exposures during and before pregnancy. Am J Obstet Gynecol 2009;200: $4-24$.

115 International Commission on Radiological Protection: Pregnancy and medical radiation. Ann ICRP 2000;30:1-43.

116 Inamasu J, Nichols TA, Guiot BH: Vertebral hemangioma symptomatic during pregnancy treated by posterior decompression, intraoperative vertebroplasty, and segmental fixation. J Spinal Disord Tech 2006;19:451454.

117 O'Sullivan GJ, Carty FL, Cronin CG: Imaging of bone metastasis: an update. World J Radiol 2015;7:202-211.

118 Torloni MR, Vedmedovska N, Merialdi M, Betrán AP, Allen T, González R, et al: Safety of ultrasonography in pregnancy: WHO systematic review of the literature and metaanalysis. Ultrasound Obstet Gynecol 2009; 33:599-608

119 Slipman CW, Sterenfeld EB, Chou LH, Her$\operatorname{zog} \mathrm{R}$, Vresilovic E: The predictive value of provocative sacroiliac joint stress maneuvers in the diagnosis of sacroiliac joint syndrome. Arch Phys Med Rehabil 1998;79: 288-292.

120 Heidari P, Farahbakhsh F, Rostami M, Noormohammadpour P, Kordi R: The role of ultrasound in diagnosis of the causes of low back pain: a review of the literature. Asian J Sports Med 2015;6:e23803.

121 Weishaupt D, Zanetti M, Hodler J, Boos N: MR imaging of the lumbar spine: prevalence of intervertebral disk extrusion and sequestration, nerve root compression, end plate abnormalities, and osteoarthritis of the facet joints in asymptomatic volunteers. Radiology 1998;209:661-666.

122 Jensen MC, Brant-Zawadzki MN, Obuchowski N, Modic MT, Malkasian D, Ross IS: Magnetic resonance imaging of the lumbar spine in people without back pain. $\mathrm{N}$ Engl J Med 1994;331:69-73.

123 Barr K: Electrodiagnosis of lumbar radiculopathy. Phys Med Rehabil Clin N Am 2013; 24:79-91.

124 Cho SC, Ferrante MA, Levin KH, Harmon RL, So YT: Utility of electrodiagnostic testing in evaluating patients with lumbosacral radiculopathy: an evidence-based review. Muscle Nerve 2010;42:276-282.

125 van Tulder MW, Assendelft WJ, Koes BW, Bouter LM: Spinal radiographic findings and nonspecific low back pain. A systematic review of observational studies. Spine (Phila Pa 1976) 1997;22:427-434.

126 Videman T, Battie MC, Gibbons LE, Maravilla $\mathrm{K}$, Manninen $\mathrm{H}$, Kaprio J: Associations between back pain history and lumbar MRI findings. Spine (Phila Pa 1976) 2003;28:582588.

127 Brinjikji W, Luetmer PH, Comstock B, Bresnahan BW, Chen LE, Deyo RA, et al: Systematic literature review of imaging features of spinal degeneration in asymptomatic populations. AJNR Am J Neuroradiol 2015;36: 811-816.

128 Hooten WM, Cohen SP: Evaluation and treatment of low back pain: a clinically focused review for primary care specialists. Mayo Clin Proc 2015;90:1699-1718.

129 National Institute for Health and Clinical Excellence: Antenatal care for uncomplicated pregnancies (CG62) [Internet]. 2008. https://www.nice.org.uk/guidance/cg62 (cited November 13, 2015).

130 Bongers PM, de Winter CR, Kompier MA, Hildebrandt VH: Psychosocial factors at work and musculoskeletal disease. Scand J Work Environ Health 1993;19:297-312.

131 Olsson CB, Grooten WJ, Nilsson-Wikmar L, Harms-Ringdahl K, Lundberg M: Catastrophizing during and after pregnancy: associations with lumbopelvic pain and postpartum physical ability. Phys Ther 2012;92:49-57.

132 Ferreira CW, Alburquerque-Sendı NF: Effectiveness of physical therapy for pregnancy-related low back and/or pelvic pain after delivery: a systematic review. Physiother Theory Pract 2013;29:419-431.

133 Pennick V, Liddle SD: Interventions for preventing and treating pelvic and back pain in pregnancy; in Pennick V (ed): Cochrane Database of Systematic Reviews. Chichester, John Wiley \& Sons, Ltd., 2013, p CD001139.

134 Zolotor AJ, Carlough MC: Update on prenatal care. Am Fam Physician 2014;89:199208.

135 Beaty CM, Bhaktaram VJ, Rayburn WF, Parker MJ, Christensen HD, Chandrasekaran K: Low backache during pregnancy. Acute hemodynamic effects of a lumbar support. J Reprod Med 1999;44:1007-1011.

136 van Duijvenbode I, Jellema P, van Poppel M, van Tulder MW: Lumbar supports for prevention and treatment of low back pain; in van Duijvenbode I (ed): Cochrane Database of Systematic Reviews. Chichester, John Wiley \& Sons, Ltd., 2008, p CD001823.

137 Cramer H, Lauche R, Haller H, Dobos G: A systematic review and meta-analysis of yoga for low back pain. Clin J Pain 2013;29:450460.

138 Combs MA, Thorn BE: Yoga attitudes in chronic low back pain: roles of catastrophizing and fear of movement. Complement Ther Clin Pract 2015;21:160-165.

139 Hill C: Is yoga an effective treatment in the management of patients with chronic low back pain compared with other care modalities - a systematic review. J Complement
Integr Med 2013;10:pii:/j/jcim.2013.10.issue-1/jcim-2012-0007/jcim-2012-0007.xml.

140 Lam M, Galvin R, Curry P: Effectiveness of acupuncture for nonspecific chronic low back pain: a systematic review and metaanalysis. Spine (Phila Pa 1976) 2013;38: 2124-2138.

141 Yuan QL, Guo TM, Liu L, Sun F, Zhang YG: Traditional Chinese medicine for neck pain and low back pain: a systematic review and meta-analysis. PLoS One 2015; 10: e0117146.

142 Zhao HJ, Tan JY, Wang T, Jin L: Auricular therapy for chronic pain management in adults: a synthesis of evidence. Complement Ther Clin Pract 2015;21:68-78.

143 Abdi S, Datta S, Trescot AM, Schultz DM, Adlaka R, Atluri SL, et al: Epidural steroids in the management of chronic spinal pain: a systematic review. Pain Physician 2007;10: 185-212.

144 Cohen SP, Bicket MC, Jamison D, Wilkinson I, Rathmell JP: Epidural steroids: a comprehensive, evidence-based review. Reg Anesth Pain Med;38:175-200.

145 Nguyen GC, Seow CH, Maxwell C, Huang $\mathrm{V}$, Leung $\mathrm{Y}$, Jones J, et al: The Toronto consensus statements for the management of inflammatory Bowel disease in pregnancy. Gastroenterology 2016;150:734-757.e1.

146 Kulkarni M, Shenoy L, Sagar MS: Anesthetic management of a pregnant patient undergoing open splenectomy for hypersplenism. Anesth Essays Res 2014;8(3):393396.

147 Cook TM, Counsell D, Wildsmith JA: Major complications of central neuraxial block: report on the Third National Audit Project of the Royal College of Anaesthetists. Br J Anaesth 2009;102:179-190.

148 Abdallah MW, Elzayyat NS, Abdelhaq MM, Gado AA: A comparative study of general anesthesia versus combined spinal - epidural anesthesia on the fetus in cesarean section. Egypt J Anaesth 2014;30:155160.

149 Saygı Aİ, Özdamar Ö, Gün İ, Emirkadı H, Müngen E, Akpak YK: Comparison of maternal and fetal outcomes among patients undergoing cesarean section under general and spinal anesthesia: a randomized clinical trial. Sao Paulo Med J 2015;133:227234.

150 Chakraverty R, Pynsent P, Isaacs K: Which spinal levels are identified by palpation of the iliac crests and the posterior superior iliac spines? J Anat 2007;210:232-236.

151 Perlas A, Chaparro LE, Chin KJ: Lumbar neuraxial ultrasound for spinal and epidural anesthesia: a systematic review and metaanalysis. Reg Anesth Pain Med 2016;41:251260.

152 Chin KJ, Perlas A, Chan V, Brown-Shreves D, Koshkin A, Vaishnav V: Ultrasound imaging facilitates spinal anesthesia in adults with difficult surface anatomic landmarks. Anesthesiology 2011;115:94-101.
LBP in Pregnancy: Role of Neuraxial Analgesia and Anaesthesia
Gynecol Obstet Invest 2017;82:417-436 DOI: $10.1159 / 000471764$ 
153 Loyola University: Ultrasound guided osterior sacroiliac ligament corticosteroid injection in pregnancy-related pelvic girdle pain; In ClinicalTrials.gov [Internet]. Bethesda, National Library of Medicine (US), 2000. https://clinicaltrials.gov/ct2/show/ NCT02044991 (cited November 16, 2015).

154 Hubbert $\mathrm{CH}$ : Epidural anesthesia in patients with spinal fusion. Anesth Analg 1985;64:843.

155 Crosby ET, Halpern SH: Obstetric epidural anaesthesia in patients with Harrington instrumentation. Can J Anaesth 1989;36:693696.

156 Daley MD, Rolbin SH, Hew EM, Morningstar BA, Stewart JA: Epidural anesthesia for obstetrics after spinal surgery. Reg Anesth 1990;15:280-284.
157 Kim SH, Jeon DH, Chang CH, Lee SJ, Shin YS: Spinal anesthesia with isobaric tetracaine in patients with previous lumbar spinal surgery. Yonsei Med J 2009;50:252-256.

158 Okutomi T, Saito M, Koura M, Hoka S: Spinal anesthesia using a continuous spinal catheter for cesarean section in a parturient with prior surgical correction of scoliosis. J Anesth 2006;20:223-226.

159 Wong CA, Scavone BM, Dugan S, Smith JC, Prather H, Ganchiff JN, et al: Incidence of postpartum lumbosacral spine and lower extremity nerve injuries. Obstet Gynecol 2003; 101:279-288.
160 Chan YL, Lam WW, Lau TK, Metreweli C, Chan DP: Back pain in pregnancy - magnetic resonance imaging correlation. Clin Radiol 2002;57:1109-1112.

161 Elden H, Ostgaard HC, Fagevik-Olsen M, Ladfors L, Hagberg H: Treatments of pelvic girdle pain in pregnant women: adverse effects of standard treatment, acupuncture and stabilising exercises on the pregnancy, mother, delivery and the fetus/neonate. BMC Complement Altern Med 2008;8:34.

162 Peterson CK, Mühlemann D, Humphreys B: Outcomes of pregnant patients with low back pain undergoing chiropractic treatment: a prospective cohort study with short term, medium term and 1 year follow-up. Chiropr Man Therap 2014;22:15. 